\title{
A Finite Element Method for a Phase Field Model of Nematic Liquid Crystal Droplets
}

\author{
Amanda E. Diegel ${ }^{1}$ and Shawn W. Walker ${ }^{1, *}$ \\ ${ }^{1}$ Department of Mathematics, Louisiana State University, Baton Rouge, \\ LA 70803-4918, USA.
}

Received 4 August 2017; Accepted (in revised version) 13 December 2017

\begin{abstract}
We develop a novel finite element method for a phase field model of nematic liquid crystal droplets. The continuous model considers a free energy comprised of three components: the Ericksen's energy for liquid crystals, the Cahn-Hilliard energy representing the interfacial energy of the droplet, and an anisotropic weak anchoring energy that enforces a condition such that the director field is aligned perpendicular to the interface of the droplet. Applications of the model are for finding minimizers of the free energy and exploring gradient flow dynamics. We present a finite element method that utilizes a special discretization of the liquid crystal elastic energy, as well as mass-lumping to discretize the coupling terms for the anisotropic surface tension part. Next, we present a discrete gradient flow method and show that it is monotone energy decreasing. Furthermore, we show that global discrete energy minimizers $\Gamma$ converge to global minimizers of the continuous energy. We conclude with numerical experiments illustrating different gradient flow dynamics, including droplet coalescence and break-up.
\end{abstract}

AMS subject classifications: 65M60, 65M12, 35Q99

Key words: Nematic liquid crystal, phase field, Ericksen's energy, $\Gamma$-convergence, gradient flow.

\section{Introduction}

The purpose of this paper is to couple Ericksen's model for nematic liquid crystals to an interfacial energy (modeled via the Cahn-Hilliard equation) in order to model liquid crystal droplets. Interest in developing numerical methods for modeling liquid crystals or complex fluids involving liquid crystals has grown in recent years, $[2,5,20,28,34,35$, $40,41,54]$. One driver for this development is the large host of technological applications of liquid crystals $[1,4,8,9,29,36,38,42,45,49,55]$. Popular models representing liquid crystal substances include the Q-tensor model, the Oseen-Frank model, and Ericksen's model with a variable degree of orientation. A common issue in any of these methods

*Corresponding author. Email addresses: diegel@math.lsu.edu (A. E. Diegel), walker@math.lsu.edu (S. W. Walker) 
is capturing defects. For instance, in [5], Barrett et al. presents a fully discrete finite element method for the evolution of uniaxial nematic liquid crystals with variable degree of orientation. An advantage of their method is that they are able to provide convergence results. However, in order to avoid the degeneracy introduced by the degree of orientation variable $s$, they use a regularization of Ericksen's model.

The use of diffuse interface theory to describe the mixing of complex fluids has likewise grown in popularity and the research group which includes J. Zhao, X. Yang, Q. Wang, J. Shen (among others) has released several papers on this subject [57-62]. Their models may be described as energy minimizing models whereby their energy functionals are composed of a kinetic energy and a free energy. The kinetic energy is based on fluid velocity coming from a fluid model, such as Stoke's flow. The free energy is then broken down into three parts: the mixing energy, the bulk free energy for liquid crystals, and an anchoring energy. For instance, in [62], Zhao et. al. develop an energy-stable scheme for a binary hydrodynamic phase field model of mixtures of nematic liquid crystals and viscous fluids where they use the Cahn-Hilliard energy to describe the mixing energy and the Oseen-Frank energy to describe the bulk free energy for liquid crystals. Defects are effectively regularized by penalizing the unit length constraint.

The work presented herein is unique in the following sense: the Cahn-Hilliard energy is combined directly with Ericksen's energy in order to develop a phase field model for nematic liquid crystal droplets in a pure liquid crystal substance. In this way, the model we present herein should be considered as a first approximation to modeling deformable colloids in liquid crystalline substances. We therefore make the assumption that the liquid crystal properties are congruent across the interface of the droplet. The model considers a free energy which is comprised of three components: the Ericksen's energy for liquid crystals, the Cahn-Hilliard energy representing the interfacial energy of the droplet, and an anisotropic weak anchoring energy that enforces a condition such that the director field is aligned perpendicular to the interface of the droplet. The goal is to find minimizers of this free energy. To this end, we present a finite element discretization of the energy and apply a modified time-discrete gradient flow method to compute minimizers. The numerical scheme considered herein combines the finite element approximation of the Ericksen model of nematic liquid crystals in [40], which captures point and line defects and requires no regularization, and the technique considered in [24] which follows a convex splitting gradient flow strategy for modeling the Cahn-Hilliard equation.

An outline of the paper is as follows. Section 2 describes the continuous energy model for the liquid crystal/surface tension system. In Section 3, we present a discretization of the total energy (2.12) followed by the development of a discrete gradient flow strategy in Section 4. In Section 5, we present a fully discrete finite element scheme based on the gradient flow strategy and prove its stability. In Section 6, we demonstrate that the discrete energy converges to the continuous energy using the tools of $\Gamma$-convergence. We conclude with several numerical experiments in Section 7, and some discussion in Section 8. 


\section{Continuous energy models}

\subsection{Ericksen energy}

We consider the one-constant model for liquid crystals with variable degree of orientation $[22,25,52]$ (Ericksen's model) on a Lipschitz domain $\Omega \subset \mathbb{R}^{d}$ with $d=2,3$. The liquid crystal state is modeled by a director field $\mathbf{n}(x)$ and a scalar function $s(x)$, the so-called degree-of-orientation. Equilibrium is attained when $(s, \mathbf{n})$ minimizes the nondimensional energy

$$
J(s, \mathbf{n})=E_{\mathrm{erk}}(s, \mathbf{n})+E_{\mathrm{dw}}(s),
$$

where $E_{\mathrm{erk}}(s, \mathbf{n})$ and $E_{\mathrm{dw}}(s)$ are defined by

$$
\begin{aligned}
& E_{\mathrm{erk}}(s, \mathbf{n}):=\int_{\Omega}\left[\kappa|\nabla s|^{2}+s^{2}|\nabla \mathbf{n}|^{2}\right] d \mathbf{x}, \\
& E_{\mathrm{dw}}(s):=\int_{\Omega} f(s(\mathbf{x})) d \mathbf{x},
\end{aligned}
$$

with $\kappa>0$ and where the double well potential $f$ is a $C^{2}$ function defined on $-1 / 2<s<1$ that satisfies the following conditions $[3,25,33]$ :

1. $\lim _{s \rightarrow 1} f(s)=\lim _{s \rightarrow-1 / 2} f(s)=\infty$,

2. $f(0)>f\left(s^{*}\right)=\min _{s \in[-1 / 2,1]} f(s)$ for some $s^{*} \in(0,1)$,

3. $f^{\prime}(0)=0$.

The existence of minimizers $\left(s^{*}, \mathbf{n}^{*}\right)$ of $(2.1)$ was shown in $[3,33]$, along with regularity properties. Minimizers may exhibit non-trivial defects (depending on boundary conditions) $[7,10,15,32,33,43]$. Some analytical solutions can be found in [52]. The presence of $s$ in (2.2) gives a degenerate Euler-Lagrange equation for $\mathbf{n}$. This allows for line and plane defects (singularities of $\mathbf{n}$ ) when $s$ vanishes in dimension $d=3$. The size of defects and regularity properties of minimizers were studied in [33]. This lead to the study of dynamics [18] and corresponding numerics [5]. However, in both cases, they regularize the model to avoid the degeneracy induced by the order parameter $s$ vanishing. In [40], they present a numerical method, without requiring any regularization, for computing minimizers of (2.1) that exhibit non-trivial defect structures.

The theoretical framework follows $[3,33]$. We introduce an auxiliary variable $\mathbf{u}:=s \mathbf{n}$, and rewrite Ericksen's energy (2.1) as

$$
E_{\text {erk }}(s, \mathbf{n})=\widetilde{E_{\text {erk }}}(s, \mathbf{u}):=\int_{\Omega}\left((\kappa-1)|\nabla s|^{2}+|\nabla \mathbf{u}|^{2}\right) d \mathbf{x},
$$

which follows from differentiating the identity $|\mathbf{n}|^{2}=1$. This suggests the following admissible class for $(s, \mathbf{u})$ :

$$
\mathbb{A}_{\text {erk }}:=\left\{(s, \mathbf{u}) \in\left[H^{1}(\Omega)\right]^{d+1}: \text { there exists } \mathbf{n} \text { such that (2.6) holds }\right\},
$$


where

$$
\mathbf{u}=s \mathbf{n}, \quad-1 / 2<s<1 \text { a.e. in } \Omega \text {, and } \mathbf{n} \in \mathbb{S}^{d-1} \text { a.e. in } \Omega,
$$

is called the structural condition of $\mathbb{A}_{\text {erk }}$. Note: we use an abuse of notation and define $(s, \mathbf{n})$ in $\mathbb{A}_{\text {erk }}$ to mean $(s, \mathbf{u})$ in $\mathbb{A}_{\text {erk }}$ with $\mathbf{u}=s \mathbf{n}$.

Moreover, to enforce boundary conditions on $(s, \mathbf{u})$, possibly on different parts of the boundary, let $\left(\Gamma_{s}, \Gamma_{\mathbf{u}}\right)$ be open subsets of $\partial \Omega$ where we set Dirichlet boundary conditions for $(s, \mathbf{u})$. Then the restricted admissible class is defined by

$$
\mathbb{A}_{\text {erk }}(g, \mathbf{r}):=\left\{(s, \mathbf{u}) \in \mathbb{A}_{\text {erk }}:\left.s\right|_{\Gamma_{s}}=g,\left.\mathbf{u}\right|_{\Gamma_{\mathbf{u}}}=\mathbf{r}\right\},
$$

for some given functions $(g, \mathbf{r}) \in\left[W_{\infty}^{1}(\Omega)\right]^{d+1}$ that satisfy (2.6) on $\partial \Omega$. If we further assume

$$
g \geq \delta_{0} \quad \text { on } \partial \Omega, \text { for some } \delta_{0}>0,
$$

then $\mathbf{n}$ is $H^{1}$ in a neighborhood of $\partial \Omega$ and satisfies $\mathbf{n}=g^{-1} \mathbf{r} \in \mathrm{S}^{d-1}$ on $\partial \Omega$.

In the case where $s$ is a non-zero constant, (2.2) effectively reduces to the Oseen-Frank energy $\int_{\Omega}|\nabla \mathbf{n}|^{2}$. If $s$ is variable, it may vanish in order to relax the energy of defects. In this case, discontinuities of $\mathbf{n}$ (i.e. defects) may occur in the singular set

$$
\mathcal{S}:=\{x \in \Omega: s(x)=0\},
$$

with finite energy: $E_{\text {erk }}(s, \mathbf{n})<\infty$. The parameter $\kappa$ in (2.2) can influence the appearance of defects; see $[39,40]$ for examples of this effect.

\subsection{Phase field energy}

The Cahn-Hilliard (CH) energy is given by $[16,17]$

$$
E_{\mathrm{ch}}(\phi)=\int_{\Omega} \frac{1}{4 \varepsilon}\left(\phi^{2}-1\right)^{2}+\frac{\varepsilon}{2} \int_{\Omega}|\nabla \phi|^{2} d \mathbf{x}=: E_{\mathrm{chdw}}(\phi)+E_{\mathrm{chp}}(\phi),
$$

where $\varepsilon>0$ is a small constant representing the interfacial width between the liquid crystal droplet and surrounding liquid crystal substance and $\phi$ represents a concentration field. The natural admissible class for $\phi$ is $H^{1}(\Omega)$. The $\mathrm{CH}$ energy (2.10) typically prefers the pure phase values $\phi= \pm 1$ and may be described as representing a competition between two different energy density terms: the double well density $\frac{1}{4 \varepsilon}\left(\phi^{2}-1\right)^{2}$ which is minimized by the pure phase values of $\phi$ and the gradient energy density $\frac{\varepsilon}{2} \int_{\Omega}|\nabla \phi|^{2}$ which penalizes any derivatives of $\phi$. Energy minimizers of (2.10) are such that the pure phases indicated by $\phi= \pm 1$ are separated by diffuse interfaces of thickness $\sim \mathcal{\varepsilon}$, such that the indicator function is essentially a hyperbolic tangent in the direction perpendicular to the interface. For example, in the one dimensional case and following the procedure presented in [27], it can be shown that minimizers of (2.10) are given as $\phi(x)= \pm \tanh \left(\frac{x-x_{0}}{\sqrt{2} \varepsilon}\right)$. In short, the $\mathrm{CH}$ energy is used as a model for motion by mean curvature. 
The interfacial energy associated with the condition that the director field is aligned perpendicular to the interface of the droplet is given by the following anisotropic "diffuse" weak anchoring energy [22,52]:

$$
\begin{aligned}
& E_{\mathrm{a}, \mathbf{n}}(s, \mathbf{n}, \phi)=\frac{\varepsilon}{2} \int_{\Omega} s^{2}\left[|\mathbf{n}|^{2}|\nabla \phi|^{2}-(\mathbf{n} \cdot \nabla \phi)^{2}\right] d \mathbf{x}, \\
& E_{\mathrm{a}, s}(s, \phi)=\frac{\varepsilon}{2} \int_{\Omega}|\nabla \phi|^{2}\left(s(\mathbf{x})-s^{*}\right)^{2} d \mathbf{x},
\end{aligned}
$$

where we note that (2.11) can be derived from [37, eqn. (66)] (the details of which can be found Appendix (8)) and where $\varepsilon$ is included to ensure that $E_{\mathrm{a}, \mathbf{n}}$ scales the same as $E_{\text {chp. }}$. The total anchoring energy is then considered to be $E_{\text {anch }}(s, \mathbf{n}, \phi):=E_{\mathrm{a}, \mathbf{n}}(s, \mathbf{n}, \phi)+$ $E_{\mathrm{a}, s}(s, \phi)$. Note that $E_{\mathrm{a}, \mathbf{n}}(\mathbf{n}, \phi)$ tries to force homeotropic anchoring of $\mathbf{n}$ on the interface of the droplet, i.e. when (2.11) is minimized, the director field $\mathbf{n}$ wants to be aligned with $\nabla \phi$ which is parallel to the normal vector of the interface. The condition that the director field $\mathbf{n}$ is perpendicular to $\nabla \phi$ (i.e. $\mathbf{n}$ is tangent to the interface), is referred to as planar anchoring, and is an obvious modification of the method presented here.

Combining the three components produces the total energy

$$
\begin{aligned}
E(s, \mathbf{n}, \phi)= & \omega_{\mathrm{erk}} E_{\mathrm{erk}}(s, \mathbf{n})+\omega_{\mathrm{dw}} E_{\mathrm{dw}}(s)+\omega_{\mathrm{chdw}} E_{\mathrm{chdw}}(\phi) \\
& +\omega_{\mathrm{chp}} E_{\mathrm{chp}}(\phi)+\omega_{\mathrm{a}, \mathbf{n}} E_{\mathrm{a}, \mathbf{n}}(s, \mathbf{n}, \phi)+\omega_{\mathrm{a}, s} E_{\mathrm{a}, s}(s, \phi),
\end{aligned}
$$

where $\omega_{\mathrm{erk}}, \omega_{\mathrm{dw}}, \omega_{\mathrm{chdw}}, \omega_{\mathrm{chp}}, \omega_{\mathrm{a}, \mathrm{n}}, \omega_{\mathrm{a}, \mathrm{s}}>0$ are constants denoting various "weights". The total energy is then described as consisting of a liquid crystal energy (using the Ericksen model), an interfacial energy (using the Cahn-Hilliard model), and an energetic coupling term that connects the two.

Remark 2.1 (Relaxation of unit length constraint). We note that as $\mathbf{n}$ is a unit vector, the 'diffuse' weak anchoring energy (2.11) could be written as

$$
E_{\mathrm{a}, \mathbf{n}}(s, \mathbf{n}, \phi)=\frac{\varepsilon}{2} \int_{\Omega} s^{2}\left[|\nabla \phi|^{2}-(\mathbf{n} \cdot \nabla \phi)^{2}\right] d \mathbf{x} .
$$

However, the condition that $\mathbf{n}$ be a unit vector will be relaxed in the discretization of the energy. See Section 3 for more details, such as (3.2). Therefore, we list the form shown in (2.11) so that the development of the discretized energy may be clearly observed.

Remark 2.2 (Anisotropic surface tension). Let $\tilde{J}(s, \mathbf{n}, \phi):=E_{\mathrm{ch}}(\phi)+E_{\mathrm{a}, \mathbf{n}}(s, \mathbf{n}, \phi)$, which has the form:

$$
\begin{aligned}
\tilde{J}(s, \mathbf{n}, \phi) & =\frac{1}{4 \varepsilon} \int_{\Omega}\left(\phi^{2}-1\right)^{2}+\frac{\varepsilon}{2} \int_{\Omega}|\nabla \phi|^{2} d \mathbf{x}+\frac{\varepsilon}{2} \int_{\Omega} s^{2}\left[|\mathbf{n}|^{2}|\nabla \phi|^{2}-(\mathbf{n} \cdot \nabla \phi)^{2}\right] d \mathbf{x} \\
& =\frac{1}{4 \varepsilon} \int_{\Omega}\left(\phi^{2}-1\right)^{2}+\frac{\varepsilon}{2} \int_{\Omega} \nabla \phi \cdot\left[\mathbf{I}+s^{2}(\mathbf{I}-\mathbf{n} \otimes \mathbf{n})\right] \nabla \phi d \mathbf{x} .
\end{aligned}
$$

Thus, combining $E_{\mathrm{ch}}$ with $E_{\mathrm{a}, \mathrm{n}}$ changes the effective surface tension from isotropic to anisotropic. We note that we have taken the weights equal to 1 for simplicity but that this property holds for any weights $\omega_{\mathrm{a}, \mathrm{n}}, \omega_{\mathrm{chdw}}, \omega_{\mathrm{chp}}>0$. 
Remark 2.3 (The limit as $\varepsilon \downarrow 0$ ). Considering Remark 2.2, it is natural to consider changes in the limiting case as $\varepsilon \downarrow 0$ relative to the anisotropic energy $\tilde{J}(s, \mathbf{n}, \phi)+E_{\mathrm{a}, s}(s, \phi)$. Though we do not offer a formal analysis here, we expect this energy to model motion by a weighted mean curvature. This is supported by the work of Taylor and Cahn in [50] and Torabi et al. in [51].

\section{Spatial discretization of the energy}

Assume the domain $\Omega$ is partitioned into a conforming simplicial triangulation $\mathcal{T}_{h}=\{K\}$. The set of nodes (vertices) of $\mathcal{T}_{h}$ is denoted $\mathcal{N}_{h}$ with cardinality $N$. We further assume the following property on the so-called stiffness matrix entries

$$
k_{i j}:=-\int_{\Omega} \nabla \eta_{i} \cdot \nabla \eta_{j} d x
$$

such that $k_{i j} \geq 0$ for all $i \neq j$ and where $\eta_{i}$ is the standard "hat" basis function associated with node $\mathbf{x}_{i} \in \mathcal{N}_{h}$. This is guaranteed if the mesh is weakly acute $[13,19,30,48]$. Note: weak acuteness is guaranteed if all interior angles (dihedral angles in three dimensions) are bounded by $90^{\circ}$; this corresponds to a non-obtuse mesh.

Next, we introduce the following finite element spaces:

$$
\begin{aligned}
& \mathbb{Y}_{h}:=\left\{\phi_{h} \in H^{1}(\Omega):\left.\phi_{h}\right|_{K} \text { is affine for all } K \in \mathcal{T}_{h}\right\}, \\
& \mathbb{S}_{h}:=\left\{s_{h} \in H^{1}(\Omega):\left.s_{h}\right|_{K} \text { is affine for all } K \in \mathcal{T}_{h}\right\}, \\
& \mathbb{U}_{h}:=\left\{\mathbf{u}_{h} \in H^{1}(\Omega)^{d}:\left.\mathbf{u}_{h}\right|_{K} \text { is affine in each component for all } K \in \mathcal{T}_{h}\right\}, \\
& \mathbb{N}_{h}:=\left\{\mathbf{n}_{h} \in \mathbb{U}_{h}:\left|\mathbf{n}_{h}\left(\mathbf{x}_{i}\right)\right|=1 \text { for all nodes } \mathbf{x}_{i} \in \mathcal{N}_{h}\right\}, \\
& \mathbb{V}_{h}^{\perp}:=\left\{\mathbf{v}_{h} \in \mathbb{U}_{h}: \mathbf{v}_{h}\left(\mathbf{x}_{i}\right) \cdot \mathbf{n}_{h}\left(\mathbf{x}_{i}\right)=0 \text { for all nodes } \mathbf{x}_{i} \in \mathcal{N}_{h}\right\},
\end{aligned}
$$

where $\mathbb{N}_{h}$ imposes the unit length constraint only at the vertices of the mesh; this implies that $\left|\mathbf{n}_{h}(\mathbf{x})\right| \leq 1$ for any $\mathbf{x} \in K$. The spaces can be modified to incorporate (Dirichlet) boundary conditions:

$$
\begin{aligned}
& \mathbb{S}_{h}\left(\Gamma_{s}, g_{h}\right):=\left\{s_{h} \in \mathbb{S}_{h}:\left.s_{h}\right|_{\Gamma_{s}}=g_{h}\right\}, \\
& \mathbb{U}_{h}\left(\Gamma_{\mathbf{u}}, \mathbf{r}_{h}\right):=\left\{\mathbf{u}_{h} \in \mathbb{U}_{h}:\left.\mathbf{u}_{h}\right|_{\Gamma_{\mathbf{u}}}=\mathbf{r}_{h}\right\},
\end{aligned}
$$

where $\Gamma_{s}, \Gamma_{\mathbf{u}}$ represent subsets of $\partial \Omega$ where Dirichlet conditions are enforced and $g_{h}=$ $I_{h} g, \mathbf{r}_{h}=I_{h} \mathbf{r}_{h}$ are the Lagrange interpolations of $(g, \mathbf{r})$ where $g$ and $\mathbf{r}$ are the traces of some $W_{\infty}^{1}(\Omega)$ functions as in (2.7). With these definitions, we define a discrete admissible class

$$
\mathbb{A}_{\text {erk }, h}\left(g_{h}, \mathbf{r}_{h}\right):=\left\{\left(s_{h}, \mathbf{u}_{h}\right) \in \mathbb{S}_{h}\left(\Gamma_{s}, g_{h}\right) \times \mathbb{U}_{h}\left(\Gamma_{\mathbf{u}}, \mathbf{r}_{h}\right): \text { there is } \mathbf{n}_{h} \text { such that (3.5) holds }\right\},
$$

where

$$
\mathbf{u}_{h}=I_{h}\left(s_{h} \mathbf{n}_{h}\right), \quad-1 / 2<s_{h}<1 \text { in } \Omega, \quad \mathbf{n}_{h} \in \mathbb{N}_{h},
$$


is the discrete structural condition of $\mathbb{A}_{\text {erk, } h}$. Again, we abuse notation and define $\left(s_{h}, \mathbf{n}_{h}\right)$ in $\mathbb{A}_{\text {erk }, h}$ to mean $\left(s_{h}, \mathbf{u}_{h}\right)$ in $\mathbb{A}_{\text {erk }, h}$ with $\mathbf{u}_{h}=I_{h}\left(s_{h} \mathbf{n}_{h}\right)$.

The discrete form of the Ericksen energy (2.2) is given by [40]

$$
E_{\text {erk }}^{h}\left(s_{h}, \mathbf{n}_{h}\right):=\frac{\kappa}{2} \sum_{i, j=1}^{N} k_{i j}\left(s_{h}\left(\mathbf{x}_{i}\right)-s_{h}\left(\mathbf{x}_{j}\right)\right)^{2}+\frac{1}{2} \sum_{i, j=1}^{N} k_{i j}\left(\frac{s_{h}\left(\mathbf{x}_{i}\right)^{2}+s_{h}\left(\mathbf{x}_{j}\right)^{2}}{2}\right)\left|\mathbf{n}_{h}\left(\mathbf{x}_{i}\right)-\mathbf{n}_{h}\left(\mathbf{x}_{j}\right)\right|^{2},
$$

for $\left(s_{h}, \mathbf{u}_{h}\right) \in \mathbb{A}_{\text {erk, } h}\left(g_{h}, \mathbf{r}_{h}\right)$ where the second term is a first order approximation of $\int_{\Omega} s^{2}|\nabla \mathbf{n}|^{2} d \mathbf{x}$. Note that it can be shown that the first term equals $\kappa \int_{\Omega}\left|\nabla s_{h}\right|^{2} d \mathbf{x}$. The discrete energy satisfies a coercivity estimate [40, Lemma 3.5] which we now summarize.

Lemma 3.1. For any $\left(s_{h}, \mathbf{n}_{h}\right) \in \mathbb{A}_{\mathrm{erk}, h}$, we have

$$
E_{\text {erk }}^{h}\left(s_{h}, \mathbf{n}_{h}\right) \geq \min \{\kappa, 1\} \max \left\{\int_{\Omega}\left|\nabla \mathbf{u}_{h}\right|^{2} d x, \int_{\Omega}\left|\nabla s_{h}\right|^{2} d \mathbf{x}\right\} \text {, where } \mathbf{u}_{h}=I_{h}\left(s_{h} \mathbf{n}_{h}\right) .
$$

The form of (3.6) is able to account for the degeneracy in $s_{h}$ in the limit as $h \rightarrow 0$ without regularization. Indeed, in [40], they proved a $\Gamma$-convergence result for (3.6), i.e.

$$
\Gamma-\lim _{h \rightarrow 0} E_{\text {erk }}^{h}\left(s_{h}, \mathbf{n}_{h}\right)=E_{\text {erk }}(s, \mathbf{n}) .
$$

The Ericksen double well energy, the Cahn-Hilliard energy, and anchoring energy $E_{\mathrm{a}, s}$ are discretized in the standard way:

$$
\begin{aligned}
& E_{\mathrm{dw}}^{h}\left(s_{h}\right):=\int_{\Omega} f\left(s_{h}(\mathbf{x})\right) d \mathbf{x}, \\
& E_{\mathrm{ch}}^{h}\left(\phi_{h}\right):=\int_{\Omega} \frac{1}{4 \varepsilon}\left(\phi_{h}^{2}-1\right)^{2}+\frac{\varepsilon}{2} \int_{\Omega}\left|\nabla \phi_{h}\right|^{2} d \mathbf{x}, \\
& E_{\mathrm{a}, s}^{h}\left(s_{h}, \phi_{h}\right)=\frac{\varepsilon}{2} \int_{\Omega}\left|\nabla \phi_{h}\right|^{2}\left(s_{h}(\mathbf{x})-s^{*}\right)^{2} d \mathbf{x} .
\end{aligned}
$$

Finally, the discrete version of the weak anchoring term $E_{\mathrm{a}, \mathbf{n}}$ is given by

$$
E_{\mathrm{a}, \mathbf{n}}\left(s_{h}, \mathbf{n}_{h}, \phi_{h}\right)=\sum_{T_{j} \subset \mathcal{T}_{h}} \int_{T_{j}} I_{h}\left\{\left(s_{h}\right)^{2} \mathbf{n}_{h} \cdot\left[\left(\nabla \phi_{h} \cdot \nabla \phi_{h}\right) \mathbf{I}-\left(\nabla \phi_{h} \otimes \nabla \phi_{h}\right)\right] \mathbf{n}_{h}\right\},
$$

where $I_{h}$ is the Lagrange interpolant. We note that a more detailed definition of the discretization of $E_{\mathrm{a}, \mathbf{n}}$ is given in Section 4.

The (total) discrete energy is then

$$
\begin{aligned}
E^{h}\left(s_{h}, \mathbf{n}_{h}, \phi_{h}\right)= & \omega_{\mathrm{erk}} E_{\mathrm{erk}}^{h}\left(s_{h}, \mathbf{n}_{h}\right)+\omega_{\mathrm{dw}} E_{\mathrm{dw}}^{h}\left(s_{h}\right)+\omega_{\mathrm{chdw}} E_{\mathrm{chdw}}^{h}\left(\phi_{h}\right) \\
& +\omega_{\mathrm{chp}} E_{\mathrm{chp}}^{h}\left(\phi_{h}\right)+\omega_{\mathrm{a}, \mathbf{n}} E_{\mathrm{a}, \mathbf{n}}^{h}\left(s_{h}, \mathbf{n}_{h}, \phi_{h}\right)+\omega_{\mathrm{a}, s} E_{\mathrm{a}, s}^{h}\left(s_{h}, \phi_{h}\right) .
\end{aligned}
$$

The discretization of time will follow a gradient flow strategy with respect to the total discrete energy (3.12). 


\section{Fully discrete gradient flow strategy}

We use the notation $(\cdot, \cdot): L^{2}(\Omega) \times L^{2}(\Omega) \rightarrow \mathbb{R}$ as the standard $L^{2}$ inner product and the notation $a(\cdot, \cdot): H^{1}(\Omega) \times H^{1}(\Omega) \rightarrow \mathbb{R}$ as the $H^{1}$ inner product such that each may be applied to both scalar and vector valued functions as follows:

$(u, v)=\int_{\Omega} u v d \mathbf{x}, \quad(\mathbf{n}, \mathbf{w})=\int_{\Omega} \mathbf{n} \cdot \mathbf{w} d \mathbf{x}, \quad a(u, v)=\int_{\Omega} \nabla u \cdot \nabla v d \mathbf{x}, \quad a(\mathbf{n}, \mathbf{w})=\int_{\Omega} \nabla \mathbf{n}: \nabla \mathbf{w} d \mathbf{x}$.

Next, we define a multi-linear form representing the discrete Ericksen's energy $E_{\text {erk }}^{h}$, as well as its variational derivatives. Specifically, we define $e(\cdot, \cdot, \cdot, \cdot): \mathbb{S}_{h} \times \mathbb{S}_{h} \times \mathbb{U}_{h} \times \mathbb{U}_{h} \rightarrow \mathbb{R}$ by

$$
\begin{aligned}
& e\left(s_{h}, z_{h} ; \mathbf{n}_{h}, \mathbf{w}_{h}\right) \\
:= & \sum_{i, j=1}^{N} k_{i j}\left(\frac{s_{h}\left(\mathbf{x}_{i}\right) z_{h}\left(\mathbf{x}_{i}\right)+s_{h}\left(\mathbf{x}_{j}\right) z_{h}\left(\mathbf{x}_{j}\right)}{2}\right)\left(\mathbf{n}_{h}\left(\mathbf{x}_{i}\right)-\mathbf{n}_{h}\left(\mathbf{x}_{j}\right)\right) \cdot\left(\mathbf{w}_{h}\left(\mathbf{x}_{i}\right)-\mathbf{w}_{h}\left(\mathbf{x}_{j}\right)\right),
\end{aligned}
$$

which is linear in each argument, and note that

$$
E_{\text {erk }}^{h}\left(s_{h}, \mathbf{n}_{h}\right)=\kappa a\left(s_{h}, z_{h}\right)+\frac{1}{2} e\left(s_{h}, s_{h} ; \mathbf{n}_{h}, \mathbf{n}_{h}\right) .
$$

Furthermore, taking variational derivatives with respect to both $s_{h}$ and $\mathbf{n}_{h}$, we have

$$
\begin{aligned}
& \delta_{\mathbf{n}_{h}} E_{\text {erk }}^{h}\left[s_{h}, \mathbf{n}_{h} ; \mathbf{w}_{h}\right]=e\left(s_{h}, s_{h} ; \mathbf{n}_{h}, \mathbf{w}_{h}\right), \\
& \delta_{s_{h}} E_{\text {erk }}^{h}\left[s_{h}, \mathbf{n}_{h} ; z_{h}\right]=2 \kappa a\left(s_{h}, z_{h}\right)+e\left(s_{h}, z_{h} ; \mathbf{n}_{h}, \mathbf{n}_{h}\right) .
\end{aligned}
$$

Additionally, the variational derivative with respect to $s_{h}$ of the Ericksen double well energy is

$$
\delta_{s_{h}} E_{\mathrm{dw}}^{h}\left(s_{h} ; z_{h}\right)=\int_{\Omega} f^{\prime}\left(s_{h}\right) z_{h} d \mathbf{x},
$$

and the variational derivative with respect to $\phi_{h}$ of the Cahn-Hilliard energy is given by

$$
\delta_{\phi_{h}} E_{\mathrm{ch}}^{h}\left(\phi_{h} ; \psi_{h}\right)=\int_{\Omega} \frac{1}{\varepsilon}\left(\phi_{h}^{3}-\phi_{h}\right) \psi_{h} d \mathbf{x}+\varepsilon a\left(\phi_{h}, \psi_{h}\right) .
$$

Finally, we define a discrete inner product to capture the discrete coupling energy $E_{\mathrm{a}, \mathbf{n}}(s, \mathbf{n}, \phi)$ in (2.11), as well as its variational derivatives. Define the multi-linear form $c(\cdot, \cdot, \cdot, \cdot ;, \cdot): \mathbb{U}_{h} \times P_{0} \times \mathbb{U}_{h} \times P_{0} \times S_{h} \times S_{h} \rightarrow \mathbb{R}$, where $P_{0}$ is the space of piecewise constant, vector-valued functions such that

$$
\begin{aligned}
& c\left(\mathbf{v}_{h}, \nabla \phi_{h}, \mathbf{w}_{h}, \nabla \psi_{h} ; s_{h}, z_{h}\right) \\
:= & \sum_{T_{j} \subset \mathcal{T}_{h}}\left|T_{j}\right| \frac{1}{d+1} \times \sum_{i=1}^{d+1}\left[\left.s_{h} z_{h}\left(\left(\nabla \phi_{h} \cdot \nabla \psi_{h}\right)\left(\mathbf{v}_{h} \cdot \mathbf{w}_{h}\right)-\left(\mathbf{v}_{h} \cdot \nabla \phi_{h}\right)\left(\mathbf{w}_{h} \cdot \nabla \psi_{h}\right)\right)\right|_{T_{j}}\left(\hat{\mathbf{x}}_{i}^{j}\right)\right],
\end{aligned}
$$


where $\left\{\hat{x}_{i}^{j}\right\}_{i=1}^{d+1}$ are the vertices of the element $T_{j}$ in the mesh $\mathcal{T}_{h}$; note that we restrict $\nabla \phi_{h}$, $\nabla \psi_{h}$ to $T_{j}$ before evaluating at $\mathbf{x}=\hat{\mathbf{x}}_{i}^{j}$. Eq. (4.6) can also be written as

$$
c\left(\mathbf{v}_{h}, \nabla \phi_{h}, \mathbf{w}_{h}, \nabla \psi_{h} ; s_{h}, z_{h}\right):=\sum_{T_{j} \subset \mathcal{T}_{h}} \int_{T_{j}} I_{h}\left\{\left(s_{h} z_{h}\right) \mathbf{v}_{h} \cdot\left[\left(\nabla \phi_{h} \cdot \nabla \psi_{h}\right) \mathbf{I}-\left(\nabla \phi_{h} \otimes \nabla \psi_{h}\right)\right] \mathbf{w}_{h}\right\},
$$

where $I_{h}$ is the Lagrange interpolant; this follows because the formula in (4.6) can be viewed as a quadrature rule that is exact for linear polynomials over each element $T_{j}$. The finite element realization of (4.6) is a $d \times d$ block matrix, where each block is an $N \times N$ diagonal matrix.

Considering these definitions, the discrete anchoring condition can be written as

$$
\begin{aligned}
& E_{\mathrm{a}, \mathbf{n}}^{h}\left(\mathbf{n}_{h}, \phi_{h}, s_{h}\right)=\frac{\varepsilon}{2} c\left(\mathbf{n}_{h}, \nabla \phi_{h}, \mathbf{n}_{h}, \nabla \phi_{h} ; s_{h}, s_{h}\right), \\
& E_{\mathrm{a}, s}^{h}\left(s_{h}, \phi_{h}\right)=\frac{\varepsilon}{2}\left(\nabla \phi_{h}\left(s_{h}(\mathbf{x})-s^{*}\right), \nabla \phi_{h}\left(s_{h}(\mathbf{x})-s^{*}\right)\right),
\end{aligned}
$$

with the following variational derivatives

$$
\begin{aligned}
& \delta_{\mathbf{n}_{h}} E_{\mathrm{a}, \mathbf{n}}^{h}\left(s_{h}, \mathbf{n}_{h}, \phi_{h} ; \mathbf{w}_{h}\right)=\varepsilon c\left(\mathbf{n}_{h}, \nabla \phi_{h}, \mathbf{w}_{h}, \nabla \phi_{h} ; s_{h}, s_{h}\right), \\
& \delta_{s_{h}} E_{\mathrm{a}, \mathbf{n}}^{h}\left(s_{h}, \mathbf{n}_{h}, \phi_{h} ; z_{h}\right)=\varepsilon c\left(\mathbf{n}_{h}, \nabla \phi_{h}, \mathbf{n}_{h}, \nabla \phi_{h} ; s_{h}, z_{h}\right), \\
& \delta_{\phi_{h}} E_{\mathrm{a}, \mathbf{n}}^{h}\left(s_{h}, \mathbf{n}_{h}, \phi_{h} ; \psi_{h}\right)=\varepsilon c\left(\mathbf{n}_{h}, \nabla \phi_{h}, \mathbf{n}_{h}, \nabla \psi_{h} ; s_{h}, s_{h}\right), \\
& \delta_{s_{h}} E_{\mathrm{a}, s}^{h}\left(s_{h}, \phi_{h} ; z_{h}\right)=\varepsilon\left(\nabla \phi_{h}\left(s_{h}-s^{*}\right), \nabla \phi_{h} z_{h}\right), \\
& \delta_{\phi_{h}} E_{\mathrm{a}, s}^{h}\left(s_{h}, \phi_{h} ; \psi_{h}\right)=\varepsilon\left(\nabla \phi_{h}\left(s_{h}-s^{*}\right), \nabla \psi_{h}\left(s_{h}-s^{*}\right)\right) .
\end{aligned}
$$

An important advantage of the inner products $e(\cdot, \cdot, \cdot, \cdot)$ and $c(\cdot, \cdot, \cdot, \cdot, \cdot, \cdot)$ is that they both satisfy a projection property with respect to $\mathbf{n}_{h}$. Specifically, we have the following lemma.

Lemma 4.1. Let e $(\cdot, \cdot, \cdot, \cdot)$ be defined by (4.1) and $c(\cdot, \cdot, \cdot, \cdot, \cdot, \cdot)$ be defined by (4.7). If $\left|\mathbf{n}_{h}\left(\mathbf{x}_{i}\right)\right| \geq 1$ at all nodes $\mathbf{x}_{i}$ in $\mathcal{N}_{h}$, then

$$
\begin{aligned}
& e\left(s_{h}, s_{h} ; \mathbf{n}_{h}, \mathbf{n}_{h}\right) \geq e\left(s_{h}, s_{h} ; \frac{\mathbf{n}_{h}}{\left|\mathbf{n}_{h}\right|}, \frac{\mathbf{n}_{h}}{\left|\mathbf{n}_{h}\right|}\right), \\
& c\left(\mathbf{n}_{h}, \nabla \phi_{h}, \mathbf{n}_{h}, \nabla \phi_{h} ; s_{h}, s_{h}\right) \geq c\left(\frac{\mathbf{n}_{h}}{\left|\mathbf{n}_{h}\right|}, \nabla \phi_{h}, \frac{\mathbf{n}_{h}}{\left|\mathbf{n}_{h}\right|}, \nabla \phi_{h} ; s_{h}, s_{h}\right) .
\end{aligned}
$$

Proof. The proof of (4.10) may be found in [40]. The proof of (4.11) follows from Proposition 4.1 (shown below) and the fact that $E_{\mathrm{a}, \mathbf{n}}^{h}\left(s_{h}, \mathbf{n}_{h}, \phi_{h}\right)=m_{h}\left(\mathbf{n}_{h}, \mathbf{n}_{h}\right)$ with $H(\mathbf{x})=\left(s_{h}\right)^{2}$ $\times\left[\left(\nabla \phi_{h} \times \nabla \phi_{h}\right) \mathbf{I}-\left(\nabla \phi_{h} \otimes \nabla \phi_{h}\right)\right]$.

Proposition 4.1 (Monotone property for lumped mass matrix). Let $m_{h}: \mathbb{N}_{h} \times \mathbb{N}_{h} \rightarrow \mathbb{R}$ be a bilinear form defined by

$$
m_{h}\left(\mathbf{n}_{h}, \mathbf{w}_{h}\right)=\sum_{T_{j} \subset \mathcal{T}_{h}} \int_{T_{j}} I_{h}\left[\mathbf{n}_{h} \cdot H(\mathbf{x}) \mathbf{w}_{h}\right] d \mathbf{x},
$$


where $H$ is a $d \times d$ symmetric positive semi-definite matrix, that is piecewise discontinuous across boundaries of mesh elements but smooth inside each element. If $\left|\mathbf{n}_{h}\left(\mathbf{x}_{i}\right)\right| \geq 1$ at all nodes $\mathbf{x}_{i}$ in $\mathcal{N}_{h}$, then

$$
m_{h}\left(\mathbf{n}_{h}, \mathbf{n}_{h}\right) \geq m_{h}\left(\frac{\mathbf{n}_{h}}{\left|\mathbf{n}_{h}\right|}, \frac{\mathbf{n}_{h}}{\left|\mathbf{n}_{h}\right|}\right)
$$

Proof. Rewrite $m_{h}\left(\mathbf{n}_{h}, \mathbf{w}_{h}\right)$ as

$$
m_{h}\left(\mathbf{n}_{h}, \mathbf{w}_{h}\right):=\sum_{T_{j} \subset \mathcal{T}_{h}}\left|T_{j}\right| \frac{1}{d+1} \sum_{i=1}^{d+1}\left[\mathbf{n}_{h}\left(\hat{\mathbf{x}}_{i}^{j}\right) \cdot H\left(\hat{\mathbf{x}}_{i}^{j}\right) \mathbf{w}_{h}\left(\hat{\mathbf{x}}_{i}^{j}\right)\right] .
$$

Then, clearly,

$$
\begin{aligned}
m_{h}\left(\mathbf{n}_{h}, \mathbf{n}_{h}\right) & =\sum_{T_{j} \subset \mathcal{T}_{h}}\left|T_{j}\right| \frac{1}{d+1} \sum_{i=1}^{d+1}\left|\mathbf{n}_{h}\left(\hat{\mathbf{x}}_{i}^{j}\right)\right|^{2}\left[\frac{\mathbf{n}_{h}\left(\hat{\mathbf{x}}_{i}^{j}\right)}{\left|\mathbf{n}_{h}\left(\hat{\mathbf{x}}_{i}^{j}\right)\right|} \cdot H\left(\hat{\mathbf{x}}_{i}^{j}\right) \frac{\mathbf{n}_{h}\left(\hat{\mathbf{x}}_{i}^{j}\right)}{\left|\mathbf{n}_{h}\left(\hat{\mathbf{x}}_{i}^{j}\right)\right|}\right] \\
& \geq \sum_{T_{j} \subset \mathcal{T}_{h}}\left|T_{j}\right| \frac{1}{d+1} \sum_{i=1}^{d+1}\left[\frac{\mathbf{n}_{h}\left(\hat{\mathbf{x}}_{i}^{j}\right)}{\left|\mathbf{n}_{h}\left(\hat{\mathbf{x}}_{i}^{j}\right)\right|} \cdot H\left(\hat{\mathbf{x}}_{i}^{j}\right) \frac{\mathbf{n}_{h}\left(\hat{\mathbf{x}}_{i}^{j}\right)}{\left|\mathbf{n}_{h}\left(\hat{\mathbf{x}}_{i}^{j}\right)\right|}\right]=m_{h}\left(\frac{\mathbf{n}_{h}}{\left|\mathbf{n}_{h}\right|}, \frac{\mathbf{n}_{h}}{\left|\mathbf{n}_{h}\right|}\right) .
\end{aligned}
$$

The proof is completed.

\section{A fully discrete numerical scheme}

To set up the numerical scheme presented below, we utilize an $L^{2}$ gradient flow strategy with respect to the director field and the orientation parameters and an $\mathrm{H}^{-1}$ gradient flow strategy with respect to the phase field parameter. We note that in order to guarantee energy stability, the time discretization is not solely based on a backward Euler method. Specifically, we use two different convex splittings for the two double well potentials and the anchoring (coupling) terms must be handled appropriately.

\subsection{Scheme}

Let $M$ be a positive integer and $0=t_{0}<t_{1}<\cdots<t_{M}=T$ be a uniform partition of $[0, T]$, with $\tau=t_{i}-t_{i-1}, i=1, \cdots, M$. The fully discrete, finite element scheme is as follows: for any $1 \leq m \leq M$, given $s_{h}^{m-1} \in \mathbb{S}_{h}, \mathbf{n}_{h}^{m-1} \in \mathbb{N}_{h}$, and $\phi_{h}^{m-1} \in \mathbb{Y}_{h}$, find $s_{h}^{m} \in \mathbb{S}_{h}, \mathbf{n}_{h}^{m} \in \mathbb{N}_{h}, \phi_{h}^{m} \in \mathbb{Y}_{h}$, and $\mu_{h}^{m} \in \mathbb{Y}_{h}$, such that

$$
\begin{aligned}
\rho\left(\mathbf{v}_{h}^{m}, \mathbf{w}_{h}\right) & +\omega_{\mathrm{erk}} e\left(s_{h}^{m-1}, s_{h}^{m-1} ; \tilde{\mathbf{n}}_{h}^{m}, \mathbf{w}_{h}\right) \\
& +\omega_{\mathrm{a}, \mathbf{n}} \varepsilon \mathcal{C}\left(\tilde{\mathbf{n}}_{h}^{m}, \nabla \phi_{h}^{m-1}, \mathbf{w}_{h}, \nabla \phi_{h}^{m-1} ; s_{h}^{m-1}, s_{h}^{m-1}\right)=0, \quad \forall \mathbf{w}_{h} \in \mathbb{V}_{h}^{\perp},
\end{aligned}
$$




$$
\begin{aligned}
\left(\delta_{\tau} s_{h}^{m}, z_{h}\right) & +\omega_{\mathrm{erk}}\left[2 \kappa a\left(s_{h}^{m}, z_{h}\right)+e\left(s_{h}^{m}, z_{h} ; \mathbf{n}_{h}^{m}, \mathbf{n}_{h}^{m}\right)\right] \\
& +\omega_{\mathrm{dw}} \delta_{s_{h}} E_{\mathrm{dw}}^{h}\left(s_{h}^{m} ; z_{h}\right)+\omega_{\mathrm{a}, s} \varepsilon\left(\left(s_{h}^{m}-s^{*}\right) \nabla \phi_{h}^{m-1}, z_{h} \nabla \phi_{h}^{m-1}\right) \\
& +\omega_{\mathrm{a}, \mathbf{n}} \varepsilon c\left(\mathbf{n}_{h}^{m}, \nabla \phi_{h}^{m-1}, \mathbf{n}_{h}^{m}, \nabla \phi_{h}^{m-1} ; \bar{s}_{h}^{m}, z_{h}\right)=0, \quad \forall z_{h} \in \mathbb{S}_{h}, \\
\left(\delta_{\tau} \phi_{h}^{m}, v_{h}\right) & +\varepsilon a\left(\mu_{h}^{m}, v_{h}\right)=0, \quad \forall v_{h} \in \mathbb{Y}_{h}, \\
\omega_{\mathrm{chdw}} \varepsilon^{-1} & \left(\left(\phi_{h}^{m}\right)^{3}-\phi_{h}^{m-1}, \psi_{h}\right)+\omega_{\mathrm{chp}} \varepsilon a\left(\phi_{h}^{m}, \psi_{h}\right)-\left(\mu_{h}^{m}, \psi_{h}\right) \\
& +\omega_{\mathrm{a}, \mathbf{n}} \varepsilon c\left(\mathbf{n}_{h}^{m}, \nabla \phi_{h}^{m}, \mathbf{n}_{h}^{m}, \nabla \psi_{h} ; s_{h}^{m}, s_{h}^{m}\right) \\
& +\omega_{\mathrm{a}, s} \varepsilon\left(\left(s_{h}^{m}-s^{*}\right) \nabla \phi_{h}^{m},\left(s_{h}^{m}-s^{*}\right) \nabla \psi_{h}\right)=0, \quad \forall \psi_{h} \in \mathbb{Y}_{h},
\end{aligned}
$$

where $\mathbb{V}_{h}^{\perp}=\mathbb{V}_{h}^{\perp}\left(\mathbf{n}_{h}^{m-1}\right), \rho>0$ is a constant, and

$$
\begin{aligned}
& \delta_{\tau} s_{h}^{m}:=\frac{s_{h}^{m}-s_{h}^{m-1}}{\tau}, \quad \delta_{\tau} \phi_{h}^{m}:=\frac{\phi_{h}^{m}-\phi_{h}^{m-1}}{\tau}, \\
& \bar{s}_{h}^{m}:=\frac{s_{h}^{m}+s_{h}^{m-1}}{2}, \quad \delta_{s_{h}} E_{\mathrm{dw}}^{h}\left(s_{h}^{m} ; z_{h}\right):=\int_{\Omega}\left[f_{c}^{\prime}\left(s_{h}^{m}\right)-f_{e}^{\prime}\left(s_{h}^{m-1}\right)\right] z_{h} d \mathbf{x}, \\
& \mathbf{v}_{h}^{m}=\delta_{\tau} \tilde{\mathbf{n}}_{h}^{m}:=\frac{\tilde{\mathbf{n}}_{h}^{m}-\mathbf{n}_{h}^{m-1}}{\tau}, \quad \text { and } \quad \mathbf{n}_{h}^{m}\left(\mathbf{x}_{i}\right):=\frac{\tilde{\mathbf{n}}_{h}^{m}\left(\mathbf{x}_{i}\right)}{\left|\tilde{\mathbf{n}}_{h}^{m}\left(\mathbf{x}_{i}\right)\right|} \text { at the nodes } \mathbf{x}_{i},
\end{aligned}
$$

such that $f_{c}, f_{e}$ are convex functions for all $s \in(-1 / 2,1)$ and $f(s)=f_{c}(s)-f_{e}(s)$. We note that the order of the method is to first solve (5.1b), normalize to compute $\mathbf{n}_{h}^{m}$ and solve (5.1c), then solve (5.1d) and (5.1e).

Due to the fact that Eqs. (5.1b)-(5.1c) are essentially uncoupled from Eqs. (5.1d)-(5.1e), then following similar arguments to what are given in [40] and [24], we have the following theorem, which we state without proof:

Theorem 5.1. For any $1 \leq m \leq M$, the fully discrete scheme (5.1b)-(5.1e) is uniquely solvable and mass conservative, i.e., $\left(\phi_{h}^{m}-\phi^{0}, 1\right)=0$.

The fully-discrete scheme (5.1b)-(5.1e) obeys the energy law stated below.

Theorem 5.2. Let $\left(\phi_{h}^{m}, \mu_{h}^{m}, \mathbf{n}_{h}^{m}, s_{h}^{m}\right) \in \mathbb{Y}_{h} \times \mathbb{Y}_{h} \times \mathbb{N}_{h} \times \mathbb{S}_{h}$ be the unique solution of (5.1b)-(5.1e), for all $1 \leq m \leq M$. Then the following energy law holds for any $h, \tau>0$ :

$$
\begin{aligned}
& E^{h}\left(s_{h}^{\ell}, \mathbf{n}_{h}^{\ell}, \phi_{h}^{\ell}\right)+\frac{\omega_{\text {erk }}}{2} \sum_{m=1}^{\ell}\left(e\left(s_{h}^{m-1}, s_{h}^{m-1} ; \tilde{\mathbf{n}}_{h}^{m}, \tilde{\mathbf{n}}_{h}^{m}\right)-e\left(s_{h}^{m-1}, s_{h}^{m-1} ; \mathbf{n}_{h}^{m}, \mathbf{n}_{h}^{m}\right)\right) \\
& +\frac{\omega_{\mathrm{a}, \mathbf{n}}}{2} \sum_{m=1}^{\ell}\left(\varepsilon c\left(\tilde{\mathbf{n}}_{h}^{m}, \nabla \phi_{h}^{m-1}, \tilde{\mathbf{n}}_{h}^{m}, \nabla \phi_{h}^{m-1} ; s_{h}^{m-1}, s_{h}^{m-1}\right)\right. \\
& \left.\quad-\varepsilon c\left(\mathbf{n}_{h}^{m}, \nabla \phi_{h}^{m-1}, \mathbf{n}_{h}^{m}, \nabla \phi_{h}^{m-1} ; s_{h}^{m-1}, s_{h}^{m-1}\right)\right) \\
& \quad+\tau \sum_{m=1}^{\ell}\left(\varepsilon\left\|\nabla \mu_{h}^{m}\right\|_{L^{2}}^{2}+\rho\left\|\delta_{\tau} \mathbf{n}_{h}^{m}\right\|_{L^{2}}^{2}+\left\|\delta_{\tau} s_{h}^{m}\right\|_{L^{2}}^{2}\right)+\omega_{\mathrm{chp}} \frac{\tau^{2}}{2} \sum_{m=1}^{\ell} \varepsilon\left\|\nabla \delta_{\tau} \phi_{h}^{m}\right\|_{L^{2}}^{2}
\end{aligned}
$$




$$
\begin{aligned}
& +\omega_{\text {chdw }} \frac{\tau^{2}}{2} \sum_{m=1}^{\ell}\left(\frac{1}{2 \varepsilon}\left\|\delta_{\tau}\left(\phi_{h}^{m}\right)^{2}\right\|_{L^{2}}^{2}+\frac{1}{\varepsilon}\left\|\phi_{h}^{m} \delta_{\tau} \phi_{h}^{m}\right\|_{L^{2}}^{2}+\frac{1}{\varepsilon}\left\|\delta_{\tau} \phi_{h}^{m}\right\|_{L^{2}}^{2}\right) \\
& +\omega_{\mathrm{erk}} \frac{\tau^{2}}{2} \sum_{m=1}^{\ell}\left(2 \kappa\left\|\nabla \delta_{\tau} s_{h}^{m}\right\|_{L^{2}}^{2}+e\left(s_{h}^{m-1}, s_{h}^{m-1} ; \delta_{\tau} \mathbf{n}_{h}^{m}, \delta_{\tau} \mathbf{n}_{h}^{m}\right)+e\left(\delta_{\tau} s_{h}^{m}, \delta_{\tau} s_{h}^{m} ; \mathbf{n}_{h}^{m}, \mathbf{n}_{h}^{m}\right)\right) \\
& +\omega_{\mathrm{a}, \mathbf{n}} \frac{\tau^{2}}{2} \sum_{m=1}^{\ell}\left(\varepsilon c\left(\mathbf{n}_{h}^{m}, \nabla \delta_{\tau} \phi_{h}^{m}, \mathbf{n}_{h}^{m}, \nabla \delta_{\tau} \phi_{h}^{m} ; s_{h}^{m}, s_{h}^{m}\right)\right. \\
& \left.\quad+\varepsilon c\left(\delta_{\tau} \mathbf{n}_{h}^{m}, \nabla \phi_{h}^{m-1}, \delta_{\tau} \mathbf{n}_{h}^{m}, \nabla \phi_{h}^{m-1} ; s_{h}^{m-1}, s_{h}^{m-1}\right)\right) \\
& +\omega_{\mathrm{a}, s} \frac{\tau^{2}}{2} \sum_{m=1}^{\ell}\left(\varepsilon\left\|\left(s_{h}^{m}-s^{*}\right) \nabla \delta_{\tau} \phi_{h}^{m}\right\|_{L^{2}}^{2}+\varepsilon\left\|\delta_{\tau} s_{h}^{m} \nabla \phi_{h}^{m}\right\|_{L^{2}}^{2}\right) \\
& \leq E^{h}\left(s_{h}^{0}, \mathbf{n}_{h}^{0}, \phi_{h}^{0}\right)
\end{aligned}
$$

for all $1 \leq \ell \leq M$ and where we note that

$$
\begin{aligned}
& e\left(s_{h}^{m-1}, s_{h}^{m-1} ; \tilde{\mathbf{n}}_{h}^{m}, \tilde{\mathbf{n}}_{h}^{m}\right)-e\left(s_{h}^{m-1}, s_{h}^{m-1} ; \mathbf{n}_{h}^{m}, \mathbf{n}_{h}^{m}\right) \geq 0, \\
& c\left(\tilde{\mathbf{n}}_{h}^{m}, \nabla \phi_{h}^{m-1}, \tilde{\mathbf{n}}_{h}^{m}, \nabla \phi_{h}^{m-1} ; s_{h}^{m-1}, s_{h}^{m-1}\right)-c\left(\mathbf{n}_{h}^{m}, \nabla \phi_{h}^{m-1}, \mathbf{n}_{h}^{m}, \nabla \phi_{h}^{m-1} ; s_{h}^{m-1}, s_{h}^{m-1}\right) \geq 0 .
\end{aligned}
$$

Moreover, the energy is monotonically decreasing, i.e.

$$
E^{h}\left(s_{h}^{\ell}, \mathbf{n}_{h}^{\ell}, \phi_{h}^{\ell}\right) \leq E^{h}\left(s_{h}^{\ell-1}, \mathbf{n}_{h}^{\ell-1}, \phi_{h}^{\ell-1}\right), \quad \text { for all } 1 \leq \ell \leq M
$$

Proof. Setting $\mathbf{w}=\mathbf{v}_{h}^{m}=\delta_{\tau} \mathbf{n}_{h}^{m}=\left(\tilde{\mathbf{n}}_{h}^{m}-\mathbf{n}_{h}^{m-1}\right) / \tau$ in (5.1b), $z_{h}=\delta_{\tau} s_{h}^{m}$ in (5.1c), $v_{h}=\mu_{h}^{m}$ in (5.1d), and $\psi_{h}=\delta_{\tau} \phi_{h}^{m}$ in (5.1e), gives

$$
\begin{aligned}
\rho\left\|\delta_{\tau} \mathbf{n}_{h}^{m}\right\|_{L^{2}}^{2} & +\omega_{\mathrm{erk}} e\left(s_{h}^{m-1}, s_{h}^{m-1} ; \tilde{\mathbf{n}}_{h}^{m}, \delta_{\tau} \mathbf{n}_{h}^{m}\right) \\
& +\omega_{\mathrm{a}, \mathbf{n}} \varepsilon \mathcal{C}\left(\tilde{\mathbf{n}}_{h}^{m}, \nabla \phi_{h}^{m-1}, \delta_{\tau} \mathbf{n}_{h}^{m}, \nabla \phi_{h}^{m-1} ; s_{h}^{m-1}, s_{h}^{m-1}\right)=0, \\
\left\|\delta_{\tau} s_{h}^{m}\right\|_{L^{2}}^{2} & +\omega_{\mathrm{erk}}\left[2 \kappa a\left(s_{h}^{m}, \delta_{\tau} s_{h}^{m}\right)+e\left(s_{h}^{m}, \delta_{\tau} s_{h}^{m} ; \mathbf{n}_{h}^{m}, \mathbf{n}_{h}^{m}\right)\right] \\
& +\omega_{\mathrm{dw}} \delta_{s_{h}} E_{\mathrm{dw}}^{h}\left(s_{h}^{m} ; \delta_{\tau} s_{h}^{m}\right)+\omega_{\mathrm{a}, s} \varepsilon\left(\left(s_{h}^{m}-s^{*}\right) \nabla \phi_{h}^{m-1}, \delta_{\tau} s_{h}^{m} \nabla \phi_{h}^{m-1}\right) \\
& +\omega_{\mathrm{a}, \mathbf{n}} \varepsilon c\left(\mathbf{n}_{h}^{m}, \nabla \phi_{h}^{m-1}, \mathbf{n}_{h}^{m}, \nabla \phi_{h}^{m-1} ; \bar{s}_{h}^{m}, \delta_{\tau} s_{h}^{m}\right)=0, \\
\frac{\omega_{\mathrm{chdw}}}{4 \varepsilon}\left(\left(\delta_{\tau} \phi_{h}^{m}, \mu_{h}^{m}\right)\right. & +\varepsilon\left\|\nabla \mu_{h}^{m}\right\|_{L^{2}}^{2}=0, \\
& \left.-\phi_{h}^{m-1}, \delta_{\tau} \phi_{h}^{m}\right)+\omega_{\mathrm{chp}} \varepsilon a\left(\phi_{h}^{m}, \delta_{\tau} \phi_{h}^{m}\right)-\left(\mu_{h}^{m}, \delta_{\tau} \phi_{h}^{m}\right) \\
& +\omega_{\mathrm{a}, \mathbf{n}} \varepsilon c\left(\mathbf{n}_{h}^{m}, \nabla \phi_{h}^{m}, \mathbf{n}_{h}^{m}, \nabla \delta_{\tau} \phi_{h}^{m} ; s_{h}^{m}, s_{h}^{m}\right) \\
& +\omega_{\mathrm{a}, s} \varepsilon\left(\left(s_{h}^{m}-s^{*}\right) \nabla \phi_{h}^{m},\left(s_{h}^{m}-s^{*}\right) \nabla \delta_{\tau} \phi_{h}^{m}\right)=0 .
\end{aligned}
$$


We note that since $(\cdot, \cdot)$ and $a(\cdot, \cdot)$ are bilinear forms and since $c(\cdot, \cdot, \cdot, \cdot, \cdot, \cdot)$ and $e(\cdot, \cdot, \cdot, \cdot)$ are multi-linear forms, we obtain the following identities:

$$
\begin{aligned}
a\left(\phi_{h}^{m}, \delta_{\tau} \phi_{h}^{m}\right)= & \frac{1}{2}\left[\delta_{\tau}\left\|\nabla \phi_{h}^{m}\right\|_{L^{2}}^{2}+\tau\left\|\nabla \delta_{\tau} \phi_{h}^{m}\right\|_{L^{2}}^{2}\right] \\
\left(\left(\phi_{h}^{m}\right)^{3}-\phi_{h}^{m-1}, \delta_{\tau} \phi_{h}^{m}\right)= & \frac{1}{4} \delta_{\tau}\left\|\left(\phi_{h}^{m}\right)^{2}-1\right\|_{L^{2}}^{2} \frac{\tau}{4}\left[\left\|\delta_{\tau}\left(\phi_{h}^{m}\right)^{2}\right\|_{L^{2}}^{2}\right. \\
& \left.+2\left\|\phi_{h}^{m} \delta_{\tau} \phi_{h}^{m}\right\|_{L^{2}}^{2}+2\left\|\delta_{\tau} \phi_{h}^{m}\right\|_{L^{2}}^{2}\right] \\
c\left(\mathbf{n}_{h}^{m}, \nabla \phi_{h}^{m}, \mathbf{n}_{h}^{m}, \nabla \delta_{\tau} \phi_{h}^{m} ; s_{h}^{m}, s_{h}^{m}\right)= & \frac{1}{2 \tau}\left(c\left(\mathbf{n}_{h}^{m}, \nabla \phi_{h}^{m}, \mathbf{n}_{h}^{m}, \nabla \phi_{h}^{m} ; s_{h}^{m}, s_{h}^{m}\right)\right. \\
& \left.-c\left(\mathbf{n}_{h}^{m}, \nabla \phi_{h}^{m-1}, \mathbf{n}_{h}^{m}, \nabla \phi_{h}^{m-1} ; s_{h}^{m}, s_{h}^{m}\right)\right) \\
& +\frac{\tau}{2} c\left(\mathbf{n}_{h}^{m}, \nabla \delta_{\tau} \phi_{h}^{m}, \mathbf{n}_{h}^{m}, \nabla \delta_{\tau} \phi_{h}^{m} ; s_{h}^{m}, s_{h}^{m}\right), \\
c\left(\tilde{\mathbf{n}}_{h}^{m}, \nabla \phi_{h}^{m-1}, \delta_{\tau} \mathbf{n}_{h}^{m}, \nabla \phi_{h}^{m-1} ; s_{h}^{m-1}, s_{h}^{m-1}\right)= & \frac{1}{2 \tau}\left(c\left(\tilde{\mathbf{n}}_{h}^{m}, \nabla \phi_{h}^{m-1}, \tilde{\mathbf{n}}_{h}^{m}, \nabla \phi_{h}^{m-1} ; s_{h}^{m-1}, s_{h}^{m-1}\right)\right. \\
& \left.-c\left(\mathbf{n}_{h}^{m-1}, \nabla \phi_{h}^{m-1}, \mathbf{n}_{h}^{m-1}, \nabla \phi_{h}^{m-1} ; s_{h}^{m-1}, s_{h}^{m-1}\right)\right) \\
& +\frac{\tau}{2} c\left(\delta_{\tau} \mathbf{n}_{h}^{m}, \nabla \phi_{h}^{m-1}, \delta_{\tau} \mathbf{n}_{h}^{m}, \nabla \phi_{h}^{m-1} ; s_{h}^{m-1}, s_{h}^{m-1}\right), \\
& +\frac{\tau}{2} e\left(s_{h}^{m-1}, s_{h}^{m-1} ; \delta_{\tau} \mathbf{n}_{h}^{m}, \delta_{\tau} \mathbf{n}_{h}^{m}\right), \\
c\left(s_{h}^{m-1}, s_{h}^{m-1} ; \tilde{\mathbf{n}}_{h}^{m}, \delta_{\tau} \mathbf{n}_{h}^{m}\right)= & \frac{1}{2 \tau}\left(e\left(s_{h}^{m-1}, s_{h}^{m-1} ; \tilde{\mathbf{n}}_{h}^{m}, \tilde{\mathbf{n}}_{h}^{m}\right)\right. \\
& \left.-e\left(s_{h}^{m-1}, s_{h}^{m-1} ; \mathbf{n}_{h}^{m-1}, \mathbf{n}_{h}^{m-1}\right)\right) \\
& -\frac{1}{2 \tau}\left\|\left(s_{h}^{m-1}-s^{*}\right) \cdot \nabla \phi_{h}^{m-1}\right\|_{L^{2}}^{2} \\
& +\frac{\tau}{2}\left\|\left(s_{h}^{m}-s^{*}\right) \cdot \nabla \delta_{\tau} \phi_{h}^{m}\right\|_{L^{2}}^{2}, \\
& \left.-c\left(\mathbf{n}_{h}^{m}, \nabla \phi_{h}^{m-1}, \mathbf{n}_{h}^{m}, \nabla \phi_{h}^{m-1} ; s_{h}^{m-1}, s_{h}^{m-1}\right)\right),(5.11) \\
\left., \mathbf{n}_{h}^{m}, \nabla \phi_{h}^{m-1} ; \bar{s}_{h}^{m}, \delta_{\tau} s_{h}^{m}\right)= & \frac{1}{2 \tau}\left(c\left(\mathbf{n}_{h}^{m}, \nabla \phi_{h}^{m-1}, \mathbf{n}_{h}^{m}, \nabla \phi_{h}^{m-1} ; s_{h}^{m}, s_{h}^{m}\right)\right. \\
\left(\left(s_{h}^{m}-s^{*}\right) \cdot \nabla \phi_{h}^{m-1}, \delta_{\tau} s_{h}^{m} \cdot \nabla \phi_{h}^{m-1}\right)= & \frac{1}{2 \tau}\left\|\left(s_{h}^{m}-s^{*}\right) \cdot \nabla \phi_{h}^{m-1}\right\|_{L^{2}}^{2} \\
\left(\left(s_{h}^{m}-s^{*}\right) \cdot \nabla \phi_{h}^{m},\left(s_{h}^{m}-s^{*}\right) \cdot \nabla \delta_{\tau} \phi_{h}^{m}\right)= & \frac{1}{2 \tau}\left\|\left(s_{h}^{m}-s^{*}\right) \cdot \nabla \phi_{h}^{m}\right\|_{L^{2}}^{2} \\
& \left.\frac{1}{2} \| s^{m}\right) \cdot \nabla \phi_{h}^{m-1} \|_{L^{2}}^{2} \\
&
\end{aligned}
$$




$$
\begin{aligned}
e\left(s_{h}^{m}, \delta_{\tau} s_{h}^{m} ; \mathbf{n}_{h}^{m}, \mathbf{n}_{h}^{m}\right)= & \frac{1}{2 \tau}\left(e\left(s_{h}^{m}, s_{h}^{m} ; \mathbf{n}_{h}^{m}, \mathbf{n}_{h}^{m}\right)\right. \\
& \left.-e\left(s_{h}^{m-1}, s_{h}^{m-1} ; \mathbf{n}_{h}^{m}, \mathbf{n}_{h}^{m}\right)\right) \\
& +\frac{\tau}{2} e\left(\delta_{\tau} s_{h}^{m}, \delta_{\tau} s_{h}^{m} ; \mathbf{n}_{h}^{m}, \mathbf{n}_{h}^{m}\right) .
\end{aligned}
$$

Additionally, following the procedures supplied in $[40,46,47,56]$, we have

$$
\int_{\Omega} f\left(s_{h}^{k+1}\right) d \mathbf{x}-\int_{\Omega} f\left(s_{h}^{k}\right) d \mathbf{x} \leq \delta_{s_{h}} E_{\mathrm{dw}}^{h}\left(s_{h}^{k+1} ; s_{h}^{k+1}-s_{h}^{k}\right),
$$

for any $s_{h}^{k}$ and $s_{h}^{k+1}$ in $S_{h}$, Therefore, combining (5.3)-(5.6), using the identities above, and applying the operator $\tau \sum_{m=1}^{\ell}$ results in (5.2).

\section{6 -}

In this section, we show that the total discrete energy (3.12) converges to the total continuous energy (2.12) in the $\Gamma$-convergence sense; this is a slightly more general result than [40, Thm 3.7] which only shows that global minimizers $\Gamma$-converge. We require the use of the following proposition whose proof may be found in [40].

Proposition 6.1. Let $\Gamma_{s}=\Gamma_{\mathbf{u}}=\partial \Omega,(s, \mathbf{u}) \in \mathbb{A}_{\text {erk }}(g, \mathbf{r})$, and let $g$ satisfy (2.8). Then, given $\delta>0$, there exists a pair $\left(s_{\delta}, \mathbf{u}_{\delta}\right) \in \mathbb{A}_{\text {erk }}(g, \mathbf{r}) \cap\left[W_{\infty}^{1}(\Omega)\right]^{d+1}$ such that

$$
\left\|(s, \mathbf{u})-\left(s_{\delta}, \mathbf{u}_{\delta}\right)\right\|_{H^{1}(\Omega)} \leq \delta .
$$

Moreover, define $\mathbf{n}_{\delta}:=\mathbf{u}_{\delta} / s_{\delta}$ if $s_{\delta} \neq 0$, and any unit vector if $s_{\delta}=0$. Then, $\mathbf{n}_{\delta}$ is Lipschitz on $\Omega \backslash\left\{\left|s_{\delta}\right| \geq \xi\right\}$, for any $\xi>0$, where the Lipschitz constant depends on $\delta$ and $\xi$.

Furthermore, in order to prove the full $\Gamma$-convergence result in Theorem 6.1, we also need the following lemma.

Lemma 6.1 (Recovery Sequence for Ericksen). Let $(s, \mathbf{u}) \in \mathbb{A}_{\text {erk }}(g, \mathbf{r})$ where $\mathbf{u}=s \mathbf{n}$ with $|\mathbf{n}|=1$ a.e. Then there exists a sequence $\left(s_{h}, \mathbf{u}_{h}\right) \in \mathbb{A}_{\text {erk, } h}\left(g_{h}, \mathbf{r}_{h}\right)$ converging to $(s, \mathbf{u})$ in $H^{1}(\Omega)$, as well as $\mathbf{n}_{h} \in \mathbb{N}_{h}$ converging to $\mathbf{n}$ in $L^{2}(\Omega \backslash \mathcal{S})$, such that

$$
E_{\text {erk }}(s, \mathbf{n})=\lim _{h \rightarrow 0} E_{\text {erk }}^{h}\left(s_{h}, \mathbf{n}_{h}\right) .
$$

Proof. First, note that we can assume $E_{\text {erk }}(s, \mathbf{n})<\infty$ (otherwise, the result is trivial). Recall from (2.4) that $E_{\text {erk }}(s, \mathbf{n})=\widetilde{E_{\text {erk }}}(s, \mathbf{u})$ when $(s, \mathbf{u}) \in \mathbb{A}_{\text {erk }}$. By Proposition 6.1, there exists $\left(s_{\delta}, \mathbf{u}_{\delta}\right) \in \mathbb{A}_{\text {erk }}(g, \mathbf{r}) \cap\left[W_{\infty}^{1}(\Omega)\right]^{d+1}$, such that $\left\|(s, \mathbf{u})-\left(s_{\delta}, \mathbf{u}_{\delta}\right)\right\|_{H^{1}(\Omega)} \rightarrow 0$, as $\delta \rightarrow 0$. Ergo, with $k>0$ being a given integer, one can choose $\delta_{k}>0$ sufficiently small so that

$$
\left\|(s, \mathbf{u})-\left(s_{\delta_{k}}, \mathbf{u}_{\delta_{k}}\right)\right\|_{H^{1}(\Omega)}<k^{-1}, \quad\left|\widetilde{E_{\text {erk }}}\left(s_{\delta_{k}}, \mathbf{u}_{\delta_{k}}\right)-\widetilde{E_{\text {erk }}}(s, \mathbf{u})\right|<C_{0} k^{-1},
$$


where the constant $C_{0}>0$ depends on $\kappa$ and $\|(s, \mathbf{u})\|_{H^{1}(\Omega)}$; in fact, the last inequality follows from the first.

Next, introduce the Lagrange interpolants $s_{h}:=I_{h}\left(s_{\delta_{k}}\right), \mathbf{u}_{h}:=I_{h}\left(\mathbf{u}_{\delta_{k}}\right)$ for some $h$ to be chosen; moreover, define

$$
\mathbf{n}_{h}\left(\mathbf{x}_{i}\right)= \begin{cases}\mathbf{u}_{h}\left(\mathbf{x}_{i}\right) / s_{h}\left(\mathbf{x}_{i}\right), & \text { if } s_{h}\left(\mathbf{x}_{i}\right) \neq 0, \\ \text { any unit vector, } & \text { otherwise, }\end{cases}
$$

for each $\mathbf{x}_{i} \in \mathcal{N}_{h}$. So, $\left(s_{h}, \mathbf{n}_{h}\right) \in \mathbb{A}_{\text {erk, } h}\left(g_{h}, \mathbf{r}_{h}\right)$. By [40, Lemma 3.3], it was shown that

$$
\lim _{h \rightarrow 0} E_{\text {erk }}^{h}\left(I_{h}(\hat{s}), I_{h}(\hat{\mathbf{n}})\right)=E_{\text {erk }}(\hat{s}, \hat{\mathbf{n}}),
$$

for all $(\hat{s}, \hat{\mathbf{u}}) \in \mathbb{A}_{\text {erk }}(g, \mathbf{r}) \cap\left[W_{\infty}^{1}(\Omega)\right]^{d+1}$, where $\hat{\mathbf{n}}$ is defined as in Proposition 6.1.

Therefore, we can choose $h_{k}<\delta_{k}$ sufficiently small so that

$$
\left\|\left(s_{\delta_{k}}, \mathbf{u}_{\delta_{k}}\right)-\left(s_{h_{k}}, \mathbf{u}_{h_{k}}\right)\right\|_{H^{1}(\Omega)}<k^{-1}, \quad\left|E_{\text {erk }}\left(s_{\delta_{k}}, \mathbf{n}_{\delta_{k}}\right)-E_{\text {erk }}^{h}\left(s_{h_{k}}, \mathbf{n}_{h_{k}}\right)\right|<k^{-1} .
$$

Combining the above, we obtain $\left|E_{\text {erk }}^{h}\left(s_{h_{k}}, \mathbf{n}_{h_{k}}\right)-E_{\text {erk }}(s, \mathbf{n})\right|<C_{1} k^{-1}$, for some constant $C_{1}$ that only depends on $\kappa$ and $\|(s, \mathbf{u})\|_{H^{1}(\Omega)}$. Thus, there exists a sequence $\left(s_{h}, \mathbf{u}_{h}\right) \in$ $\mathbb{A}_{\text {erk, } h}\left(g_{h}, \mathbf{r}_{h}\right)$ converging to $(s, \mathbf{u})$ in $H^{1}(\Omega)$, as well as $\mathbf{n}_{h} \in \mathbb{N}_{h}$ converging to $\mathbf{n}$ in $L^{2}(\Omega \backslash$ $\mathcal{S})$, such that $\lim _{h \rightarrow 0} E_{\text {erk }}^{h}\left(s_{h}, \mathbf{n}_{h}\right)=E_{\text {erk }}(s, \mathbf{n})$.

We are now in position to prove the main convergence result. Since the droplets are assumed to have a fixed mass, we introduce the following constrained spaces:

$$
H_{c}^{1}(\Omega)=\left\{\phi \in H^{1}(\Omega): \int_{\Omega} \phi=c\right\}, \quad \mathbb{Y}_{h, c}=\left\{\phi_{h} \in \mathbb{Y}_{h}: \int_{\Omega} \phi_{h}=c\right\} .
$$

The discrete energy $E^{h}\left(s_{h}, \mathbf{n}_{h}, \phi_{h}\right)$ is defined on $\mathbb{W}_{h}:=\mathbb{S}_{h} \times \mathbb{N}_{h} \times \mathbb{Y}_{h, c}$, but convergence cannot be insured for a sequence $\left(s_{h}, \mathbf{n}_{h}, \phi_{h}\right) \in \mathbb{W}_{h}$, because $\mathbf{n}_{h}$ will not (in general) converge on the singular set $\mathcal{S}$. However, we can guarantee convergence for $\left(s_{h}, \mathbf{u}_{h}, \phi_{h}\right) \in \mathbb{X}_{h}:=$ $\mathrm{S}_{h} \times \mathbb{U}_{h} \times \mathbb{Y}_{h, c}$, i.e. $\mathbf{u}_{h}$ is well-behaved. Thus, Theorem 6.1 does not follow the standard definition of $\Gamma$-convergence $[11,21]$ but is similar; indeed, one level of indirection is used in stating the convergence.

To this end, we define the continuous space to be $\mathbb{X}:=L^{2}(\Omega) \times\left[L^{2}(\Omega)\right]^{d} \times L^{2}(\Omega)$, and note that $\mathbb{X}_{h} \subset \mathbb{X}$ and $\mathbb{W}_{h} \subset \mathbb{X}$. Furthermore, we define $\mathbb{A}:=\mathbb{A}_{\text {erk }}(g, \mathbf{r}) \times H_{c}^{1}(\Omega)$ and $\mathbb{A}_{h}:=$ $\mathbb{A}_{\text {erk }, h}\left(g_{h}, \mathbf{r}_{h}\right) \times \mathbb{Y}_{h, c}$. Next, the continuous energy $E: \mathbb{X} \rightarrow \mathbb{R}$ is defined as follows: $E(s, \mathbf{n}, \phi)$ by $(2.12)$ if $(s, \mathbf{n}, \phi) \in \mathbb{A}$, and set $E(s, \mathbf{n}, \phi)=\infty$ if $(s, \mathbf{n}, \phi) \in \mathbb{X} \backslash \mathbb{A}$. Likewise, define the discrete energy $E^{h}\left(s_{h}, \mathbf{n}_{h}, \phi_{h}\right)$ by (3.12) if $\left(s_{h}, \mathbf{n}_{h}, \phi_{h}\right) \in \mathbb{A}_{h}$, and set $E^{h}(s, \mathbf{n}, \phi)=\infty$ if $(s, \mathbf{n}, \phi) \in \mathbb{X} \backslash \mathbb{A}_{h}$.

Theorem 6.1 ( $\Gamma$-convergence). Given $(s, \mathbf{n}, \phi) \in \mathbb{X}$, where $|\mathbf{n}|=1$ a.e., define the corresponding element $(s, \mathbf{u}, \phi) \in \mathbb{X}$, where $\mathbf{u}:=s \mathbf{n}$. In addition, given $\left(s_{h}, \mathbf{n}_{h}, \phi_{h}\right) \in \mathbb{W}_{h}$, define the corresponding element $\left(s_{h}, \mathbf{u}_{h}, \phi_{h}\right) \in \mathbb{X}_{h}$, where $\mathbf{u}_{h}:=I_{h}\left(s_{h} \mathbf{n}_{h}\right)$. Let $\left\{\mathcal{T}_{h}\right\}$ be a sequence of weakly acute meshes and let $\gamma_{0}>0$ be some arbitrary fixed constant. Then the following properties hold for any triple $(s, \mathbf{n}, \phi)$ in $\mathbb{X}$, where $|\mathbf{n}|=1$ a.e. and $-1 / 2+\gamma_{0} \leq s \leq 1-\gamma_{0}$ a.e. 
- Lim-inf inequality. For every sequence $\left(s_{h}, \mathbf{n}_{h}, \phi_{h}\right) \in \mathbb{W}_{h} \subset \mathbb{X}$, such that the corresponding sequence $\left(s_{h}, \mathbf{u}_{h}, \phi_{h}\right) \in \mathbb{X}_{h} \subset \mathbb{X}$ converges strongly to the corresponding triple $(s, \mathbf{u}, \phi)$, we have

$$
E(s, \mathbf{n}, \phi) \leq \liminf _{h \rightarrow 0} E^{h}\left(s_{h}, \mathbf{n}_{h}, \phi_{h}\right) ;
$$

- Lim-sup inequality. There exists a sequence $\left(s_{h}, \mathbf{n}_{h}, \phi_{h}\right) \in \mathbb{W}_{h} \subset \mathbb{X}$ such that the corresponding sequence $\left(s_{h}, \mathbf{u}_{h}, \phi_{h}\right) \in \mathbb{X}_{h} \subset \mathbb{X}$ converges strongly to the corresponding triple $(s, \mathbf{u}, \phi)$, and

$$
E(s, \mathbf{n}, \phi) \geq \limsup _{h \rightarrow 0} E^{h}\left(s_{h}, \mathbf{n}_{h}, \phi_{h}\right) .
$$

Proof. The proof is split into two parts.

Part 1: Lim-inf inequality.

Let $\left(s_{h}, \mathbf{n}_{h}, \phi_{h}\right) \in \mathbb{W}_{h}$ be any sequence such that its corresponding sequence $\left(s_{h}, \mathbf{u}_{h}, \phi_{h}\right)$ $\in \mathbb{X}_{h}$ converges strongly to $(s, \mathbf{u}, \phi) \in \mathbb{X}$. Ergo, by hypothesis, we have

$$
\begin{array}{lll}
s_{h} \rightarrow s \text { in } L^{2}(\Omega), & \mathbf{u}_{h} \rightarrow \mathbf{u} \text { in } L^{2}(\Omega), & \phi_{h} \rightarrow \phi \text { in } L^{2}(\Omega), \\
s_{h} \rightarrow s \text { a.e. in } \Omega, & \mathbf{u}_{h} \rightarrow \mathbf{u} \text { a.e. in } \Omega, & \phi_{h} \rightarrow \phi \text { a.e. in } \Omega .
\end{array}
$$

Without loss of generality, we can assume that $E(s, \mathbf{n}, \phi)<\infty$; note: this implies that $(s, \mathbf{n}) \in \mathbb{A}_{\text {erk }}(g, \mathbf{r})$. Moreover, we can assume there exists a constant $\Lambda>0$ such that

$$
\begin{aligned}
\liminf _{h \rightarrow 0} E^{h}\left(s_{h}, \mathbf{n}_{h}, \phi_{h}\right)= & \liminf _{h \rightarrow 0}\left(\omega_{\mathrm{erk}} E_{\mathrm{erk}}^{h}\left(s_{h}, \mathbf{n}_{h}\right)+\omega_{\mathrm{dw}} E_{\mathrm{dw}}^{h}\left(s_{h}\right)+\omega_{\mathrm{chdw}} E_{\mathrm{chdw}}^{h}\left(\phi_{h}\right)\right. \\
& \left.+\omega_{\mathrm{chp}} E_{\mathrm{chp}}^{h}\left(\phi_{h}\right)+\omega_{\mathrm{a}, \mathbf{n}} E_{\mathrm{a}, \mathbf{n}}^{h}\left(s_{h}, \mathbf{n}_{h}, \phi_{h}\right)+\omega_{\mathrm{a}, s} E_{\mathrm{a}, \mathrm{s}}^{h}\left(s_{h}, \phi_{h}\right)\right) \leq \Lambda
\end{aligned}
$$

otherwise, the inequality (6.2) is trivial. Assumption (6.4) also implies that $\left(s_{h}, \mathbf{n}_{h}\right) \in$ $\mathbb{A}_{\text {erk }, h}\left(g_{h}, \mathbf{r}_{h}\right)$ for $h$ sufficiently small. Combining (6.4) with Lemma 3.1 (coercivity) gives the following weakly convergent subsequences (not relabeled):

$$
s_{h} \rightarrow s \text { in } H^{1}(\Omega), \quad \mathbf{u}_{h} \rightarrow \mathbf{u} \text { in } H^{1}(\Omega), \quad \phi_{h} \rightarrow \phi \text { in } H^{1}(\Omega) .
$$

Note: if $E(s, \mathbf{n}, \phi)=\infty$, then either $(s, \mathbf{n}) \notin \mathbb{A}_{\text {erk }}(g, \mathbf{r})$ or $\phi \notin H^{1}(\Omega)$. In the later case, clearly $\liminf _{h \rightarrow 0} E^{h}\left(s_{h}, \mathbf{n}_{h}, \phi_{h}\right)=\infty$, which contradicts (6.4). For the former, either $s \notin$ $H^{1}(\Omega)$ or $\mathbf{u} \notin\left[H^{1}(\Omega)\right]^{d}$. Again, this implies $\liminf _{h \rightarrow 0} E^{h}\left(s_{h}, \mathbf{n}_{h}, \phi_{h}\right)=\infty$, which contradicts (6.4). Therefore, if $E(s, \mathbf{n}, \phi)=\infty$, then the inequality (6.2) is trivial.

Using Fatou's lemma, one can show that $E_{\mathrm{dw}}(s) \leq \liminf _{h \rightarrow 0} E_{\mathrm{dw}}^{h}\left(s_{h}\right)$. In [40], the following technical result was proved: $E_{\text {erk }}(s, \mathbf{n}) \leq \liminf _{h \rightarrow 0} E_{\text {erk }}^{h}\left(s_{h}, \mathbf{n}_{h}\right)$; so we do not repeat the argument here. We now consider the remaining terms. By weak lower semicontinuity, we have

$$
E_{\mathrm{chp}}(\phi)=\frac{\varepsilon}{2} \int_{\Omega}|\nabla \phi|^{2} \leq \liminf _{h \rightarrow 0} \frac{\varepsilon}{2} \int_{\Omega}\left|\nabla \phi_{h}\right|^{2}=\liminf _{h \rightarrow 0} E_{\mathrm{chp}}^{h}\left(\phi_{h}\right) .
$$


Additionally, using the compact Sobolev embedding $H^{1}(\Omega) \hookrightarrow L^{4}(\Omega)$, for $d=2,3$, there exists a subsequence $\left\{\phi_{h}\right\}$ (not relabeled) such that $\phi_{h} \rightarrow \phi$ in $L^{4}(\Omega)$. The lim-inf inequality relating to $E_{\mathrm{chdw}}(\phi)$ then follows from Fatou's Lemma:

$$
E_{\mathrm{chdw}}(\phi)=\frac{1}{4 \varepsilon} \int_{\Omega}\left(\phi^{2}-1\right)^{2} \leq \liminf _{h \rightarrow 0} \frac{1}{4 \varepsilon} \int_{\Omega}\left(\phi_{h}^{2}-1\right)^{2}=\liminf _{h \rightarrow 0} E_{\mathrm{chdw}}^{h}\left(\phi_{h}\right) .
$$

For the anchoring energy $E_{\mathrm{a}, s}^{h}\left(s_{h}, \phi_{h}\right)$, we split the integral into two parts by adding and subtracting appropriate terms as follows:

$$
\begin{aligned}
E_{\mathrm{a}, \mathrm{s}}^{h}\left(s_{h}, \phi_{h}\right) & =\frac{\varepsilon}{2} \int_{\Omega}\left|\nabla \phi_{h}\right|^{2}\left(s_{h}-s^{*}\right)^{2} \\
& =\frac{\varepsilon}{2} \int_{\Omega}\left|\nabla \phi_{h}\right|^{2}\left(s-s^{*}\right)^{2}+\frac{\varepsilon}{2} \int_{\Omega}\left|\nabla \phi_{h}\right|^{2}\left[\left(s_{h}-s^{*}\right)^{2}-\left(s-s^{*}\right)^{2}\right] .
\end{aligned}
$$

By Egorov's theorem, given $\delta>0$, there exists a subset $A_{\delta} \subset \Omega$ such that $\left(s_{h}-s^{*}\right)^{2} \rightarrow\left(s-s^{*}\right)^{2}$ uniformly on $A_{\delta}$ and $\left|\Omega \backslash A_{\delta}\right| \leq \delta$. Hence,

$$
\begin{aligned}
\left.\lim _{h \rightarrow 0}\left|\int_{A_{\delta}}\right| \nabla \phi_{h}\right|^{2}\left[\left(s_{h}-s^{*}\right)^{2}-\left(s-s^{*}\right)^{2}\right] \mid & \leq \lim _{h \rightarrow 0}\left\|\left(s_{h}-s^{*}\right)^{2}-\left(s-s^{*}\right)^{2}\right\|_{L^{\infty}\left(A_{\delta}\right)} \int_{A_{\delta}}\left|\nabla \phi_{h}\right|^{2} \\
& \leq \lim _{h \rightarrow 0}\left\|\left(s_{h}-s^{*}\right)^{2}-\left(s-s^{*}\right)^{2}\right\|_{L^{\infty}\left(A_{\delta}\right)} \int_{\Omega}\left|\nabla \phi_{h}\right|^{2} \\
& \leq C \Lambda \lim _{h \rightarrow 0}\left\|\left(s_{h}-s^{*}\right)^{2}-\left(s-s^{*}\right)^{2}\right\|_{L^{\infty}\left(A_{\delta}\right)} \\
& =0 .
\end{aligned}
$$

Thus,

$$
\begin{aligned}
& \liminf _{h \rightarrow 0} \int_{\Omega}\left|\nabla \phi_{h}\right|^{2}\left(s_{h}-s^{*}\right)^{2} \\
\geq & \liminf _{h \rightarrow 0} \int_{A_{\delta}}\left|\nabla \phi_{h}\right|^{2}\left(s_{h}-s^{*}\right)^{2} \\
= & \liminf _{h \rightarrow 0} \int_{A_{\delta}}\left|\nabla \phi_{h}\right|^{2}\left(s-s^{*}\right)^{2}+\liminf _{h \rightarrow 0} \int_{A_{\delta}}\left|\nabla \phi_{h}\right|^{2}\left[\left(s_{h}-s^{*}\right)^{2}-\left(s-s^{*}\right)^{2}\right] \\
\geq & \liminf _{h \rightarrow 0} \int_{A_{\delta}}\left|\nabla \phi_{h}\right|^{2}\left(s-s^{*}\right)^{2} \\
\geq & \int_{A_{\delta}}|\nabla \phi|^{2}\left(s-s^{*}\right)^{2},
\end{aligned}
$$

for all $\delta>0$, where we have used weak lower semi-continuity [26]. Using Lebesgue's dominated convergence theorem and allowing $\delta \rightarrow 0$ gives the desired result.

To show the lim-inf inequality for the weak anchoring energy $E_{\mathrm{a}, \mathbf{n}}$, we begin by noting that, by using the same notation defined in Section 2 and the auxiliary variable $\mathbf{u}:=s \mathbf{n}$, the weak anchoring energy $E_{\mathrm{a}, \mathbf{n}}$ can be rewritten as:

$$
E_{\mathrm{a}, \mathbf{n}}(s, \mathbf{n}, \phi)=E_{\mathrm{a}, \mathbf{n}}(\mathbf{u}, \phi)=\frac{\varepsilon}{2} \int_{\Omega}|\mathbf{u}|^{2}|\nabla \phi|^{2}-(\mathbf{u} \cdot \nabla \phi)^{2} .
$$


Furthermore, we consider the discrete weak anchoring energy in the form of (4.7) and note the following equivalences:

$$
\begin{aligned}
E_{\mathrm{a}, \mathbf{n}}^{h}\left(s_{h}, \mathbf{n}_{h}, \phi_{h}\right) & :=\frac{\varepsilon}{2} \sum_{T_{j} \subset \mathcal{T}_{h}} \int_{T_{j}} I_{h}\left\{s_{h}^{2} \mathbf{n}_{h} \cdot\left[\left(\nabla \phi_{h} \cdot \nabla \phi_{h}\right) \mathbf{I}-\left(\nabla \phi_{h} \otimes \nabla \phi_{h}\right)\right] \mathbf{n}_{h}\right\} \\
& =\frac{\varepsilon}{2} \sum_{T_{j} \subset \mathcal{T}_{h}} \int_{T_{j}} I_{h}\left\{\left|\mathbf{u}_{h}\right|^{2}\left|\nabla \phi_{h}\right|^{2}\right\}-I_{h}\left\{\left(\nabla \phi_{h} \cdot \mathbf{u}_{h}\right)^{2}\right\}=: E_{\mathrm{a}, \mathbf{n}}^{h}\left(\mathbf{u}_{h}, \phi_{h}\right) .
\end{aligned}
$$

By interpolation theory, we have

$$
\left\|I_{h}\left\{\left|\mathbf{u}_{h}\right|^{2}\right\}-\left|\mathbf{u}_{h}\right|^{2}\right\|_{L^{2}(\Omega)} \leq C h\left\|\nabla \mathbf{u}_{h}\left|\mathbf{u}_{h}\right|\right\|_{L^{2}(\Omega)} \leq C h\left\|\nabla \mathbf{u}_{h}\right\|_{L^{2}(\Omega)} \leq C \Lambda^{1 / 2} h
$$

Similarly,

$$
\left\|I_{h}\left\{\mathbf{u}_{h} \otimes \mathbf{u}_{h}\right\}-\mathbf{u}_{h} \otimes \mathbf{u}_{h}\right\|_{L^{2}(\Omega)} \leq C \Lambda^{1 / 2} h
$$

Therefore, since $\mathbf{u}_{h} \rightarrow \mathbf{u}$ in $L^{2}(\Omega)$, we have the following convergence results,

$$
\begin{aligned}
& \left.\left|I_{h}\left\{\left|\mathbf{u}_{h}\right|^{2}\right\}-\right| \mathbf{u}\right|^{2} \mid \rightarrow 0, \quad \text { in } L^{2}(\Omega),\left.\quad\left|I_{h}\left\{\left|\mathbf{u}_{h}\right|^{2}\right\}-\right| \mathbf{u}\right|^{2} \mid \rightarrow 0, \quad \text { a.e. in } \Omega \text {, } \\
& \left|I_{h}\left\{\mathbf{u}_{h} \otimes \mathbf{u}_{h}\right\}-\mathbf{u} \otimes \mathbf{u}\right| \rightarrow 0 \text {, in } L^{2}(\Omega), \quad\left|I_{h}\left\{\mathbf{u}_{h} \otimes \mathbf{u}_{h}\right\}-\mathbf{u} \otimes \mathbf{u}\right| \rightarrow 0 \text {, a.e. in } \Omega \text {. }
\end{aligned}
$$

Due to the fact that $\nabla \phi_{h}$ is constant on each element, the discrete energy $E_{\mathrm{a}, \mathbf{n}}^{h}$ can be written as follows:

$$
\begin{aligned}
E_{\mathrm{a}, \mathbf{n}}^{h}\left(\mathbf{u}_{h}, \phi_{h}\right)= & \frac{\varepsilon}{2} \int_{\Omega}\left|\nabla \phi_{h}\right|^{2} I_{h}\left\{\left|\mathbf{u}_{h}\right|^{2}\right\}-\nabla \phi_{h} \cdot\left(I_{h}\left\{\mathbf{u}_{h} \otimes \mathbf{u}_{h}\right\}\right) \nabla \phi_{h} \\
= & \frac{\varepsilon}{2} \int_{\Omega}\left|\nabla \phi_{h}\right|^{2}|\mathbf{u}|^{2}-\left(\nabla \phi_{h} \cdot \mathbf{u}\right)^{2}+\frac{\varepsilon}{2} \int_{\Omega}\left|\nabla \phi_{h}\right|^{2}\left[I_{h}\left\{\left|\mathbf{u}_{h}\right|^{2}\right\}-|\mathbf{u}|^{2}\right] \\
& -\frac{\varepsilon}{2} \int_{\Omega} \nabla \phi_{h} \cdot\left[I_{h}\left\{\mathbf{u}_{h} \otimes \mathbf{u}_{h}\right\}-\mathbf{u} \otimes \mathbf{u}\right] \nabla \phi_{h} .
\end{aligned}
$$

By Egorov's theorem, given $\delta>0$, there exists a subset $A_{\delta} \subset \Omega$ such that $I_{h}\left\{\left|\mathbf{u}_{h}\right|^{2}\right\} \rightarrow|\mathbf{u}|^{2}$ uniformly on $A_{\delta}$ and $\left|\Omega \backslash A_{\delta}\right| \leq \delta$. Hence,

$$
\begin{aligned}
\left.\lim _{h \rightarrow 0}\left|\int_{A_{\delta}}\right| \nabla \phi_{h}\right|^{2}\left[I_{h}\left\{\left|\mathbf{u}_{h}\right|^{2}\right\}-|\mathbf{u}|^{2}\right] \mid & \leq \lim _{h \rightarrow 0}\left\|I_{h}\left\{\left|\mathbf{u}_{h}\right|^{2}\right\}-|\mathbf{u}|^{2}\right\|_{L^{\infty}\left(A_{\delta}\right)} \int_{A_{\delta}}\left|\nabla \phi_{h}\right|^{2} \\
& \leq \lim _{h \rightarrow 0}\left\|I_{h}\left\{\left|\mathbf{u}_{h}\right|^{2}\right\}-|\mathbf{u}|^{2}\right\|_{L^{\infty}\left(A_{\delta}\right)} \int_{\Omega}\left|\nabla \phi_{h}\right|^{2} \\
& \leq C \Lambda \lim _{h \rightarrow 0}\left\|I_{h}\left\{\left|\mathbf{u}_{h}\right|^{2}\right\}-|\mathbf{u}|^{2}\right\|_{L^{\infty}\left(A_{\delta}\right)} \\
& =0 .
\end{aligned}
$$


Similarly, there exists a subset $\tilde{A}_{\delta} \subset \Omega$ such that $I_{h}\left\{\mathbf{u}_{h} \otimes \mathbf{u}_{h}\right\} \rightarrow \mathbf{u} \otimes \mathbf{u}$ uniformly on $\tilde{A}_{\delta}$ and $\left|\Omega \backslash \tilde{A}_{\delta}\right| \leq \delta$. Hence

$$
\begin{aligned}
\lim _{h \rightarrow 0}\left|\int_{\tilde{A}_{\delta}} \nabla \phi_{h} \cdot\left[\mathbf{u} \otimes \mathbf{u}-I_{h}\left\{\mathbf{u}_{h} \otimes \mathbf{u}_{h}\right\}\right] \nabla \phi_{h}\right| & \leq \lim _{h \rightarrow 0}\left\|\mathbf{u} \otimes \mathbf{u}-I_{h}\left\{\mathbf{u}_{h} \otimes \mathbf{u}_{h}\right\}\right\|_{L^{\infty}\left(\tilde{A}_{\delta}\right)} \int_{\tilde{A}_{\delta}}\left|\nabla \phi_{h}\right|^{2} \\
& \leq \lim _{h \rightarrow 0}\left\|\mathbf{u} \otimes \mathbf{u}-I_{h}\left\{\mathbf{u}_{h} \otimes \mathbf{u}_{h}\right\}\right\|_{L^{\infty}\left(\tilde{A}_{\delta}\right)} \int_{\Omega}\left|\nabla \phi_{h}\right|^{2} \\
& \leq C \Lambda \lim _{h \rightarrow 0}\left\|\mathbf{u} \otimes \mathbf{u}-I_{h}\left\{\mathbf{u}_{h} \otimes \mathbf{u}_{h}\right\}\right\|_{L^{\infty}\left(\tilde{A}_{\delta}\right)} \\
& =0 .
\end{aligned}
$$

Let $B_{\delta}:=A_{\delta} \cap \tilde{A}_{\delta}$. Then $\left|\Omega \backslash B_{\delta}\right|=\left|\left(\Omega \backslash A_{\delta}\right) \cup\left(\Omega \backslash \tilde{A}_{\delta}\right)\right| \leq 2 \delta$. Hence,

$$
\begin{aligned}
& \liminf _{h \rightarrow 0} \int_{\Omega} I_{h}\left\{\left|\mathbf{u}_{h}\right|^{2}\left|\nabla \phi_{h}\right|^{2}-\left(\nabla \phi_{h} \cdot \mathbf{u}_{h}\right)^{2}\right\} \\
\geq & \liminf _{h \rightarrow 0} \int_{B_{\delta}} I_{h}\left\{\left|\mathbf{u}_{h}\right|^{2}\left|\nabla \phi_{h}\right|^{2}-\left(\nabla \phi_{h} \cdot \mathbf{u}_{h}\right)^{2}\right\} \\
= & \liminf _{h \rightarrow 0} \int_{B_{\delta}}\left|\nabla \phi_{h}\right|^{2}|\mathbf{u}|^{2}-\left(\nabla \phi_{h} \cdot \mathbf{u}\right)^{2}+\liminf _{h \rightarrow 0} \int_{B_{\delta}}\left|\nabla \phi_{h}\right|^{2}\left[I_{h}\left\{\left|\mathbf{u}_{h}\right|^{2}\right\}-|\mathbf{u}|^{2}\right] \\
& -\liminf _{h \rightarrow 0} \int_{B_{\delta}} \nabla \phi_{h} \cdot\left[I_{h}\left\{\mathbf{u}_{h} \otimes \mathbf{u}_{h}\right\}-\mathbf{u} \otimes \mathbf{u}\right] \nabla \phi_{h} \\
\geq & \liminf _{h \rightarrow 0} \int_{B_{\delta}}\left|\nabla \phi_{h}\right|^{2}|\mathbf{u}|^{2}-\left(\nabla \phi_{h} \cdot \mathbf{u}\right)^{2} \\
\geq & \int_{B_{\delta}}|\nabla \phi|^{2}|\mathbf{u}|^{2}-(\nabla \phi \cdot \mathbf{u})^{2},
\end{aligned}
$$

for all $\delta>0$, where we have used weak lower semi-continuity [26]. Using Lebesgue's dominated convergence theorem and allowing $\delta \rightarrow 0$ gives the desired result.

\section{Part 2: Lim-sup inequality.}

For the lim-sup inequality, we will construct a sequence that verifies the inequality (6.3). Indeed, we will actually show equality with a limit.

Invoking Lemma 6.1, there exists sequences $\left(s_{h}, \mathbf{u}_{h}\right) \in \mathbb{A}_{\text {erk, } h}\left(g_{h}, \mathbf{r}_{h}\right)$ and $\mathbf{n}_{h} \in \mathbb{N}_{h}$ such that

$$
\left\|\left(s_{h}, \mathbf{u}_{h}\right)-(s, \mathbf{u})\right\|_{H^{1}(\Omega)} \rightarrow 0, \quad\left\|\mathbf{n}_{h}-\mathbf{n}\right\|_{L^{2}(\Omega \backslash \mathcal{S})} \rightarrow 0,
$$

and $E_{\text {erk }}(s, \mathbf{n})=\lim _{h \rightarrow 0} E_{\text {erk }}^{h}\left(s_{h}, \mathbf{n}_{h}\right)$. For the Ericksen double-well, $E_{\mathrm{dw}}(s)$, since $-1 / 2+$ $\gamma_{0} \leq s \leq 1-\gamma_{0},|f(s(\mathbf{x}))| \leq M$ for a.e. $\mathbf{x} \in \Omega$ for some positive constant $M$ (recall Section 2.1). Thus, by Lebesgue's dominated convergence theorem, we have $\lim _{h \rightarrow 0} E_{\mathrm{dw}}^{h}\left(s_{h}\right)=E_{\mathrm{dw}}(s)$. We shall use the sequence $\left(s_{h}, \mathbf{u}_{h}\right)$ below to prove convergence of the weak anchoring terms.

For the phase variable $\phi \in H_{c}^{1}(\Omega)$, we let $\phi_{h}$ be the elliptic projection of $\phi$, i.e. $\phi_{h}$ solves $\left(\nabla \phi_{h}, \nabla \psi_{h}\right)=\left(\nabla \phi, \nabla \psi_{h}\right)$, for all $\psi_{h} \in \mathbb{Y}_{h}$, such that $\int_{\Omega} \phi_{h}=\int_{\Omega} \phi$, 
which implies that $\phi_{h} \in \mathbb{Y}_{h, c}$ and $\left\|\phi_{h}-\phi\right\|_{H^{1}(\Omega)} \rightarrow 0$. Considering

$$
E_{\mathrm{ch}}(\phi)-E_{\mathrm{ch}}\left(\phi_{h}\right)=\int_{\Omega} \frac{\omega_{\mathrm{chdw}}}{4 \varepsilon}\left[\left(\phi^{2}-1\right)^{2}-\left(\phi_{h}^{2}-1\right)^{2}\right]+\frac{\omega_{\mathrm{chp}} \varepsilon}{2}\left[|\nabla \phi|^{2}-\left|\nabla \phi_{h}\right|^{2}\right],
$$

we see that $E_{\mathrm{ch}}\left(\phi_{h}\right) \rightarrow E_{\mathrm{ch}}(\phi)$, where we used the Sobolev embedding $H^{1}(\Omega) \hookrightarrow L^{4}(\Omega)$, for $d=2,3$.

Next, since $\left(s_{h}-s^{*}\right)^{2} \rightarrow\left(s-s^{*}\right)^{2}$ a.e. in $\Omega$, and $s$ is bounded a.e. in $\Omega$, then $|\nabla \phi|^{2}\left(s_{h}-\right.$ $\left.s^{*}\right)^{2} \rightarrow|\nabla \phi|^{2}\left(s-s^{*}\right)^{2}$ a.e. in $\Omega$. So, by Lebesgue's Dominated Convergence theorem, we have

$$
\begin{aligned}
& \lim _{h \rightarrow 0}\left|E_{\mathrm{a}, s}(s, \phi)-E_{\mathrm{a}, \mathrm{s}}\left(s_{h}, \phi_{h}\right)\right| \\
= & \left.\lim _{h \rightarrow 0} \frac{\varepsilon}{2} \int_{\Omega}|| \nabla \phi\right|^{2}-\left.\left|\nabla \phi_{h}\right|^{2}\left|\left(s_{h}-s^{*}\right)^{2}+\frac{\varepsilon}{2} \int_{\Omega}\right| \nabla \phi\right|^{2}\left|\left(s-s^{*}\right)^{2}-\left(s_{h}-s^{*}\right)^{2}\right| \\
\leq & \left.\lim _{h \rightarrow 0} \frac{\varepsilon}{2} \int_{\Omega}|| \nabla \phi\right|^{2}-\left.\left|\nabla \phi_{h}\right|^{2}\left|\left(s_{h}-s^{*}\right)^{2}+\lim _{h \rightarrow 0} \frac{\varepsilon}{2} \int_{\Omega}\right| \nabla \phi\right|^{2}\left|\left(s-s^{*}\right)^{2}-\left(s_{h}-s^{*}\right)^{2}\right| \\
\leq & \left.\lim _{h \rightarrow 0} \frac{C \varepsilon}{2} \int_{\Omega}|| \nabla \phi\right|^{2}-\left|\nabla \phi_{h}\right|^{2} \mid+0=0 .
\end{aligned}
$$

Similarly, we find that

$$
\begin{aligned}
& \quad \lim _{h \rightarrow 0}\left|E_{\mathrm{a}, \mathbf{n}}(\mathbf{u}, \phi)-E_{\mathrm{a}, \mathbf{n}}^{h}\left(\mathbf{u}_{h}, \phi_{h}\right)\right| \\
& \leq\left.\lim _{h \rightarrow 0} \frac{\varepsilon}{2} \int_{\Omega}|| \nabla \phi\right|^{2}-\left.\left|\nabla \phi_{h}\right|^{2}|| \mathbf{u}\right|^{2}+\left.\lim _{h \rightarrow 0} \frac{\varepsilon}{2} \int_{\Omega}\left|\nabla \phi_{h}\right|^{2}|| \mathbf{u}\right|^{2}-I_{h}\left\{\left|\mathbf{u}_{h}\right|^{2}\right\} \mid \\
& \quad+\lim _{h \rightarrow 0} \frac{\varepsilon}{2} \int_{\Omega}\left|\nabla \phi+\nabla \phi_{h}\right||\mathbf{u} \otimes \mathbf{u}|\left|\nabla \phi-\nabla \phi_{h}\right|+\lim _{h \rightarrow 0} \frac{\varepsilon}{2} \int_{\Omega}\left|\nabla \phi_{h}\right|\left|\mathbf{u} \otimes \mathbf{u}-I_{h}\left\{\mathbf{u}_{h} \otimes \mathbf{u}_{h}\right\}\right|\left|\nabla \phi_{h}\right| \\
& =0,
\end{aligned}
$$

where one can show by interpolation theory (c.f. proof of the liminf) that

$$
\left\|I_{h}\left\{\left|\mathbf{u}_{h}\right|^{2}\right\}-\left|\mathbf{u}_{h}\right|^{2}\right\|_{L^{\infty}(\Omega)}=\mathcal{O}(h), \quad\left\|I_{h}\left\{\mathbf{u}_{h} \otimes \mathbf{u}_{h}\right\}-\mathbf{u}_{h} \otimes \mathbf{u}_{h}\right\|_{L^{\infty}(\Omega)}=\mathcal{O}(h) .
$$

The proof is completed. 21].

We now obtain the following corollary about convergence of global minimizers [12,

Corollary 6.1 (Convergence of global discrete minimizers). Let $\left\{\mathcal{T}_{h}\right\}$ be a sequence of weakly acute meshes. If $\left(s_{h}, \mathbf{n}_{h}, \phi_{h}\right) \in \mathbb{A}_{h}$ is a sequence of global minimizers of $E^{h}\left(s_{h}, \mathbf{n}_{h}, \phi_{h}\right)$ in (3.12), then every cluster point is a global minimizer of the continuous energy $E(s, \mathbf{n}, \phi)$ in (2.12).

Proof. First note that, because of the form of the energy (both continuous and discrete), we can always truncate $s$ and $s_{h}$ with the function

$$
\Theta(f):=\max \left\{-1 / 2+\gamma_{0}, \min \left\{1-\gamma_{0}, f\right\}\right\},
$$


for some fixed constant $\gamma_{0}>0$ sufficiently small. Since the boundary condition $g$ (for $s$ ) is bounded away from $-1 / 2$ and 1 (recall (2.6) and (2.8)), one can show that

$$
\begin{array}{ll}
E_{\text {erk }}(\Theta(s), \mathbf{n}) \leq E_{\text {erk }}(s, \mathbf{n}), & E_{\mathrm{dw}}(\Theta(s)) \leq E_{\mathrm{dw}}(s), \\
E_{\mathrm{a}, \mathbf{n}}(\Theta(s), \mathbf{n}, \phi) \leq E_{\mathrm{a}, \mathbf{n}}(s, \mathbf{n}, \phi), & E_{\mathrm{a}, s}(\Theta(s), \phi) \leq E_{\mathrm{a}, s}(s, \phi),
\end{array}
$$

where we use the fact that $\left(\Theta(s)-s^{*}\right)^{2} \leq\left(s-s^{*}\right)^{2}$ provided $s^{*}$ is bounded away from $-1 / 2$ and 1 . The same holds for the discrete energies as well. Thus, without loss of generality, we assume the discrete minimizers obey $-1 / 2+\gamma_{0} \leq s_{h} \leq 1-\gamma_{0}$ for $\gamma_{0}$ sufficiently small.

Next, we take $E^{h}\left(s_{h}, \mathbf{n}_{h}, \phi_{h}\right) \leq \Lambda$ for all $h>0$, where $0<\Lambda<\infty$ is a fixed constant. Using [40, Lem 3.6] we obtain convergent subsequences $\left\{s_{h}\right\},\left\{\mathbf{u}_{h}\right\},\left\{\phi_{h}\right\}$ (not relabeled) such that

$$
\begin{aligned}
& s_{h} \rightarrow s \text { in } H^{1}(\Omega), \quad \mathbf{u}_{h} \rightarrow \mathbf{u} \text { in } H^{1}(\Omega), \phi_{h} \rightarrow \phi \text { in } H_{c}^{1}(\Omega), \\
& s_{h} \rightarrow s \text { in } L^{2}(\Omega), \quad \mathbf{u}_{h} \rightarrow \mathbf{u} \text { in } L^{2}(\Omega), \quad \phi_{h} \rightarrow \phi \text { in } L^{2}(\Omega), \\
& s_{h} \rightarrow s \text { a.e. in } \Omega, \quad \mathbf{u}_{h} \rightarrow \mathbf{u} \text { a.e. in } \Omega, \phi_{h} \rightarrow \phi \text { a.e. in } \Omega .
\end{aligned}
$$

Moreover, [40, Lem 3.6] implies there is a subsequence $\left\{\mathbf{n}_{h}\right\}$ (not relabeled), and $\mathbf{n} \in L^{2}(\Omega)$ with $|\mathbf{n}|=1$ a.e., such that $\mathbb{N}_{h} \ni \mathbf{n}_{h} \rightarrow \mathbf{n}$ in $L^{2}(\Omega \backslash \mathcal{S}), \mathbf{n}_{h} \rightarrow \mathbf{n}$ a.e. in $\Omega \backslash \mathcal{S}$, and $\mathbf{u}=s \mathbf{n}$ a.e. in $\Omega$. Thus, $(s, \mathbf{u}) \in \mathbb{A}_{\text {erk }}(g, \mathbf{r})$. So the subsequence $\left(s_{h}, \mathbf{u}_{h}, \phi_{h}\right)$ of minimizers converges to a limit in $\mathbb{X}$.

Therefore, $(s, \mathbf{n}, \phi)$ and the corresponding $(s, \mathbf{u}, \phi)$ satisfies the conditions of Theorem 6.1, so we obtain that $E(s, \mathbf{n}, \phi) \leq \liminf _{h \rightarrow 0} E^{h}\left(s_{h}, \mathbf{n}_{h}, \phi_{h}\right)$. Moreover, there exists a sequence $\left\{\left(\tilde{s}_{h}, \tilde{\mathbf{n}}_{h}, \tilde{\phi}_{h}\right)\right\}$, and corresponding sequence $\left\{\left(\tilde{s}_{h}, \tilde{\mathbf{u}}_{h}, \tilde{\phi}_{h}\right)\right\}$ such that $\left(\tilde{s}_{h}, \tilde{\mathbf{u}}_{h}, \tilde{\phi}_{h}\right) \rightarrow$ $(s, \mathbf{u}, \phi)$ in $\mathbb{X}$, and

$$
E(s, \mathbf{n}, \phi) \leq \liminf _{h \rightarrow 0} E^{h}\left(s_{h}, \mathbf{n}_{h}, \phi_{h}\right) \leq \limsup _{h \rightarrow 0} E^{h}\left(\tilde{s}_{h}, \tilde{\mathbf{n}}_{h}, \tilde{\phi}_{h}\right) \leq E(s, \mathbf{n}, \phi) .
$$

Hence, $E(s, \mathbf{n}, \phi)=\lim _{h \rightarrow 0} E^{h}\left(s_{h}, \mathbf{n}_{h}, \phi_{h}\right)$, i.e. the limit of discrete global minimizers is a global minimizer.

Note: this convergence result does not yield a rate of convergence, though first order is expected in most situations (see [41] for an example).

\section{Numerical experiments}

In this section, we present numerical experiments to demonstrate our method. For the interpretation of the simulations, we note that one can have a liquid crystal droplet inside another liquid crystal droplet [31,44]. Furthermore, one can have colloids with homeotropic weak anchoring in physical experiments and the manipulation of these colloids through the use of boundary conditions is a topic of interest. Indeed, it is possible to create soft colloidal particles that contain liquid crystal material. Thus, one can view the 
two-phase simulations presented here with one phase acting as a deformable colloidal particle with homeotropic anchoring conditions imposed on its interface. We refer the interested reader to $[31,44]$ and the references therein.

In the experiments to follow, we use a square domain $\Omega=(0,1)^{2} \subset \mathbb{R}^{2}$ and take $\mathcal{T}_{h}$ to be a regular triangulation of $\Omega$ consisting of right isosceles triangles. (We note that the analysis presented in the previous sections holds for both $d=2$ and $d=3$.) To solve the linear systems in (5.1b)-(5.1c), we used MATLAB's "backslash" command and used a standard Newton's method algorithm to solve the system (5.1d)-(5.1e) with a tolerance of $10^{-15}$ or a residual tolerance of $10^{-7}$, whichever is satisfied first. To solve the linear system within Newton's method, we again used MATLAB's "backslash" command. In each experiment, the double well potential related to the orientation parameter is defined as $f(s)=f_{c}(s)-f_{e}(s)=63.0 s^{2}-\left(-16.0 s^{4}+21.33333333333 s^{3}+57.0 s^{2}\right)$ with $s^{*}=0.750025$. All computations are completed using the FELICITY MATLAB/C++ Toolbox [53] (more information on FELICITY can be found in a recently submitted paper by the second author).

\subsection{Movement of a liquid crystal droplet}

The first numerical experiment demonstrates the movement of a liquid crystal droplet. The movement of the droplet is enforced by the choice of a degree one boundary condition imposed on the director field. Specifically, we choose boundary conditions on the director field such that a defect at $(0.85,0.85)$ will eventually be observed. However, the initial conditions are such that a defect is observed at the center of the colloid at a position of $(0.26,0.25)$. Therefore, the droplet moves in order to place the defect (inside) in a more optimal location with respect to the elastic energy of the liquid crystal. Note that the defect stays inside the droplet because it is advantageous with respect to the (diffuse) weak anchoring condition on the interface. In other words, homeotropic anchoring on the droplet interface prefers a defect at the droplet center.

The initial conditions are as follows:

$$
\begin{aligned}
& s_{h}^{0}=s^{*}, \quad \mathbf{n}_{h}^{0}=\frac{(x, y)-(0.26,0.25)}{|(x, y)-(0.26,0.25)|} \\
& \phi_{h}^{0}=I_{h}\left\{-\tanh \left(\frac{(x-0.25)^{2} / 0.02+(y-0.25)^{2} / 0.02-1}{2 \varepsilon}\right)\right\} .
\end{aligned}
$$

The following Dirichlet boundary conditions on $\partial \Omega$ are imposed for $s$ and $\mathbf{n}$ :

$$
s=s^{*}, \quad \mathbf{n}_{h}=\frac{(x, y)-(0.85,0.85)}{|(x, y)-(0.85,0.85)|} .
$$

The relevant parameters are $\kappa=1, \rho=1, \omega_{\text {erk }}=1, \omega_{\text {dw }}=100, \omega_{\text {chdw }}=1, \omega_{\text {chp }}=1+$ $\omega_{\mathrm{a}, \mathbf{n}}+\omega_{\mathrm{a}, \mathrm{s}}=41, \omega_{\mathrm{a}, \mathrm{s}}=20, \omega_{\mathrm{a}, \mathbf{n}}=20$. The space step size is taken to be $h=1 / 64$ and the time step size is taken to be $\tau=0.002$ with a final stopping time of $T=20.0$. The interfacial 

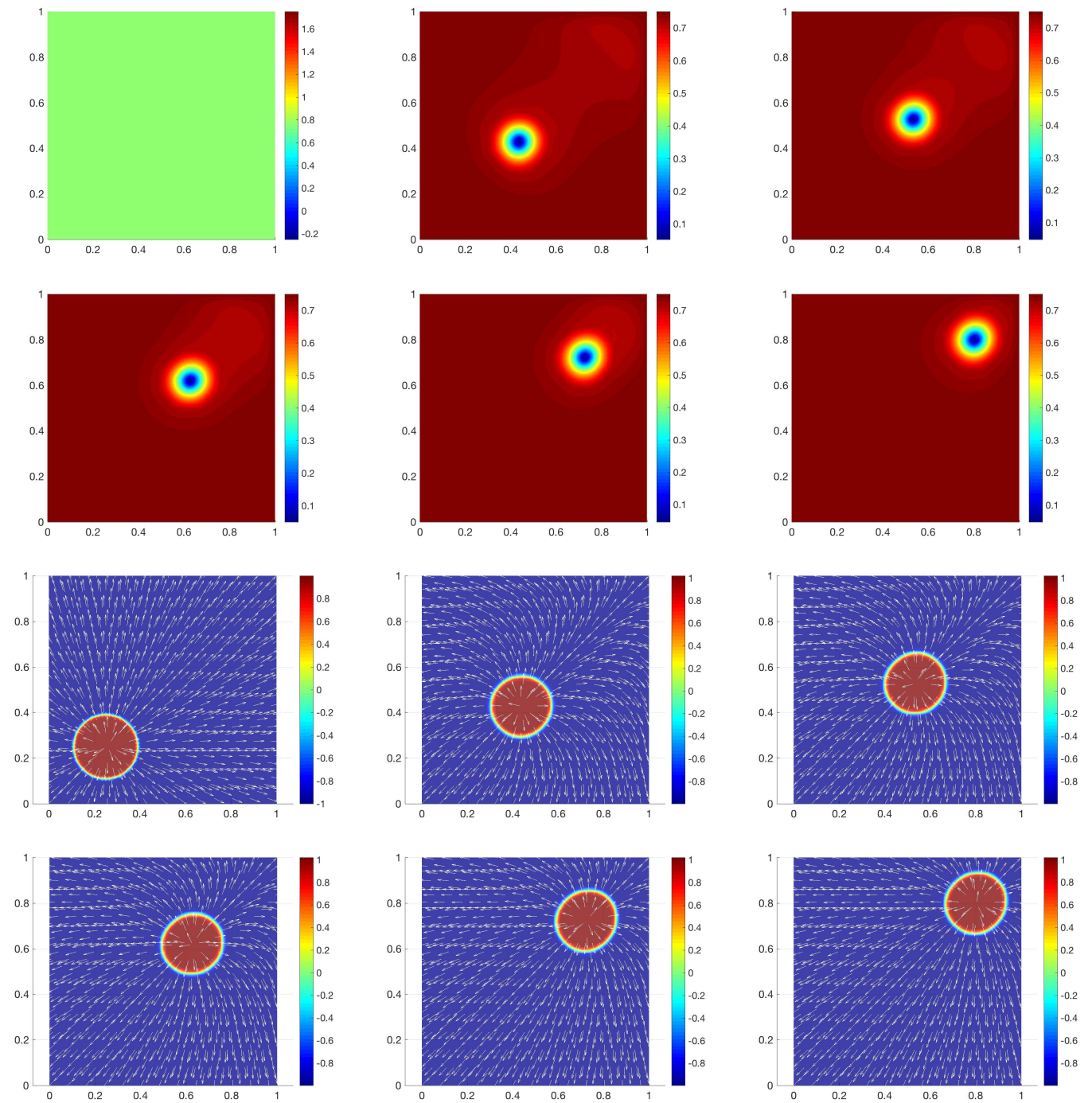

Figure 1: Droplet moving, $\Omega=[0,1] \times[0,1], h=1 / 64, \tau=0.002$ (Section 7.1). The times displayed are $t=0, t=$ $4, t=8$ (top from left to right) and $t=12, t=16, t=20$ (bottom from left to right).

width parameter is taken to be $\varepsilon=3 h$. Fig. 1 shows the evolution of the droplet over time. The top two rows display the evolution of the scalar degree of orientation parameter $s$. The bottom two rows show the evolution of the phase field parameter $\phi$ and the director field $\mathbf{n}$. This example shows that the droplet position can be manipulated by choosing appropriate boundary conditions.

Fig. 2 displays the energy decreasing property of the scheme for this experiment. We point out that the energy decreases dramatically at the beginning of the simulation due to the droplet adjusting to its equilibrium shape but then levels off. 

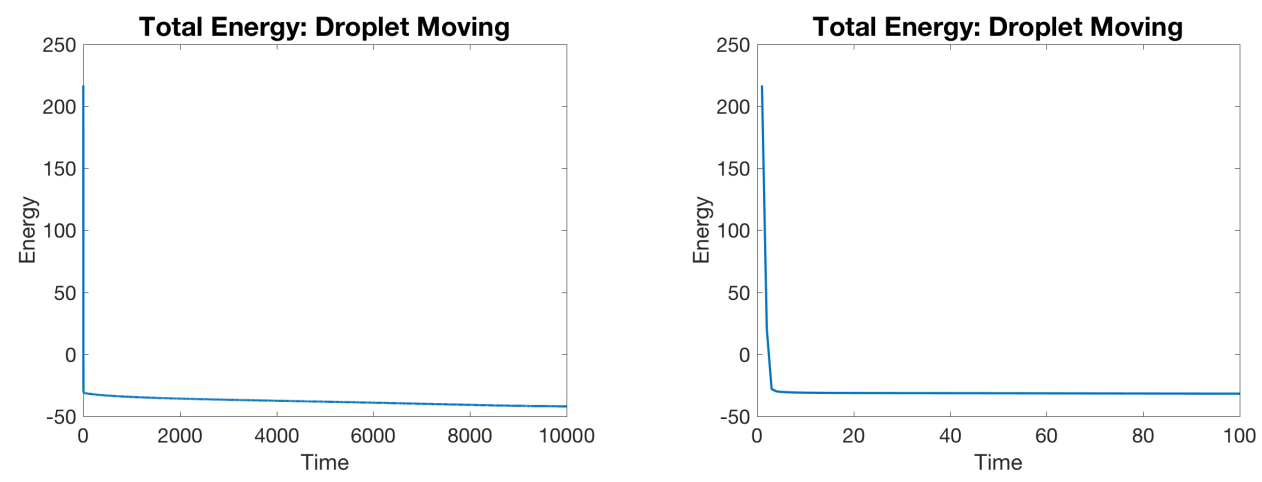

Figure 2: Total energy as a function of time for a moving droplet (Section 7.1).

\subsection{Cornering effect of a liquid crystal droplet}

The second numerical experiment demonstrates the "cornering" effect of a liquid crystal droplet. The cornering effect on liquid crystal droplets is readily observed in physical experiments [44] and the references therein. Again, the dynamics of the droplet results from the choice of boundary conditions on the director field. Specifically, degree zero (no defect) boundary conditions are chosen for the director field on the outer boundary.

Since the droplet interface has homeotropic anchoring conditions imposed, it is desirable for the droplet interface normal vector and director field to be parallel. This naturally creates a competition between the interface normal and the director field. For the constants chose in this example, both the droplet shape and director field change to accommodate each other, which leads to an elongated droplet with "corners".

In contrast, choosing $\omega_{\text {erk }}$ very large would keep $\mathbf{n}$ nearly constant, which would have forced the droplet to deform significantly so that $\nabla \phi$ is nearly parallel to $\mathbf{n}$. Alternatively, choosing $\omega_{\text {chdw }}, \omega_{\text {chp }}$ very large would have favored a droplet with isotropic surface tension, so it would prefer to remain in a (near) circular shape. If $\omega_{\mathrm{a}, \mathbf{n}}$ is also large (but $\omega_{\text {erk }}$ small), then $\mathbf{n}$ would vary significantly from a constant field to accommodate the droplet interface shape.

The initial conditions are as follows:

$$
\begin{aligned}
& s_{h}^{0}=s^{*}, \quad \mathbf{n}_{h}^{0}=(1,0), \\
& \phi_{h}^{0}=I_{h}\left\{-\tanh \left(\frac{(x-0.5)^{2} / 0.02+(y-0.5)^{2} / 0.02-1}{2 \varepsilon}\right)\right\} .
\end{aligned}
$$

The following Dirichlet boundary conditions on $\partial \Omega$ are imposed for $s$ and $\mathbf{n}$ :

$$
s=s^{*}, \quad \mathbf{n}_{h}=(1,0) .
$$

The relevant parameters are $\kappa=1, \rho=1, \omega_{\text {erk }}=1, \omega_{\text {dw }}=100, \omega_{\text {chdw }}=1, \omega_{\text {chp }}=1+\omega_{\mathrm{a}, \mathbf{n}}+$ $\omega_{\mathrm{a}, s}=41, \omega_{\mathrm{a}, s}=20, \omega_{\mathrm{a}, \mathbf{n}}=20$. The space step size is taken to be $h=1 / 64$ and the time step 

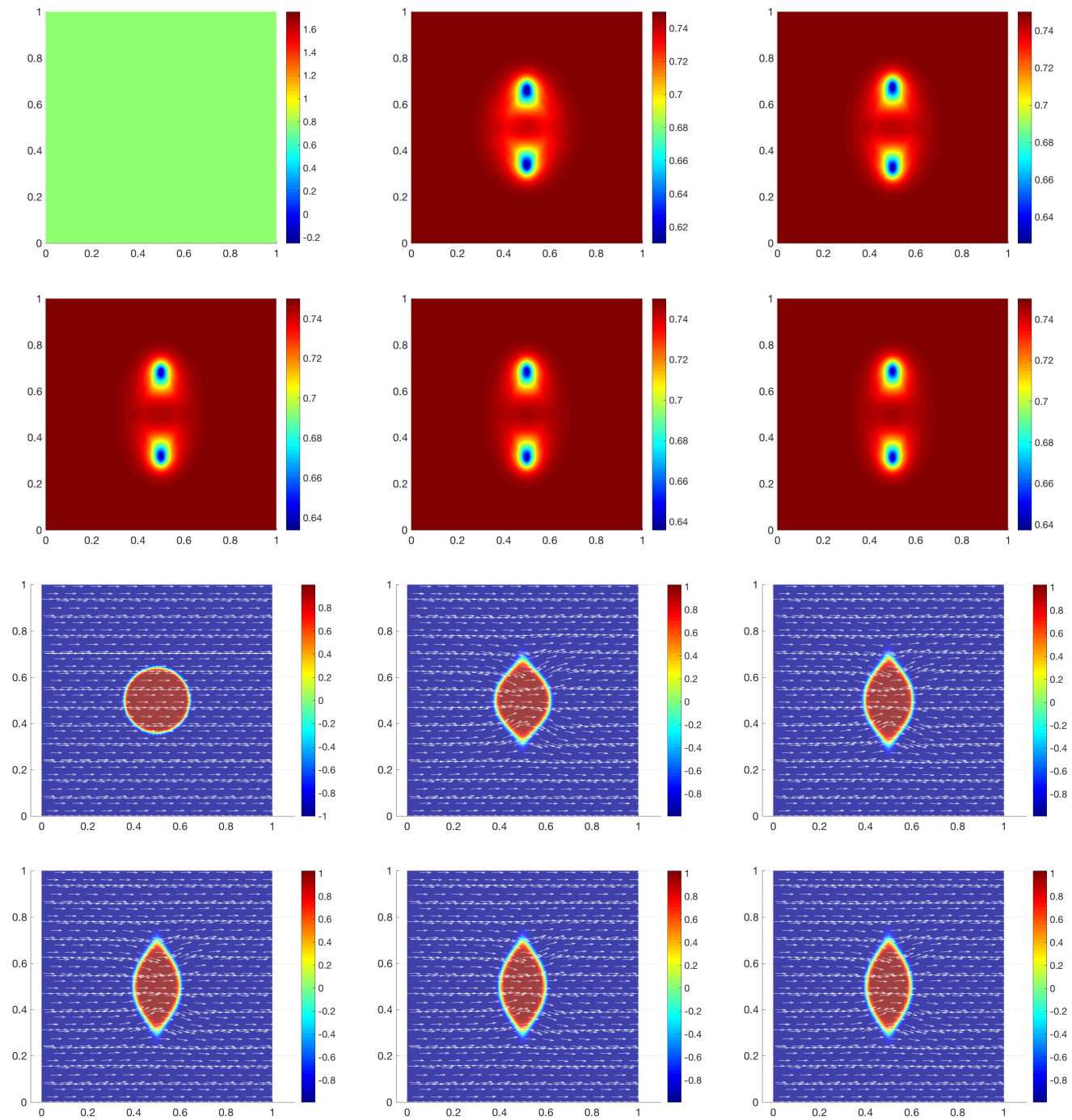

Figure 3: Droplet cornering, $\Omega=[0,1] \times[0,1], h=1 / 64, \tau=0.002$ (Section 7.2). The times displayed are $t=0$, $t=0.04, t=0.08$ (top from left to right) and $t=0.12, t=0.16, t=0.2$ (bottom from left to right).

size is taken to be $\tau=0.002$ with a final stopping time of $T=2.0$. The interfacial width parameter is taken to be $\varepsilon=3 h$. Fig. 3 shows the evolution of the droplet over time. The top two rows display the evolution of the scalar degree of orientation parameter $s$. The bottom two rows show the evolution of the phase field parameter $\phi$ and the director field n. The droplet takes on a "lens" shape with corners at the top and bottom. Note that the cornering is not sharp due to having finite surface tension, as well as a finite interfacial width parameter $\varepsilon$.

Fig. 4 displays the energy decreasing property of the scheme for this experiment. 

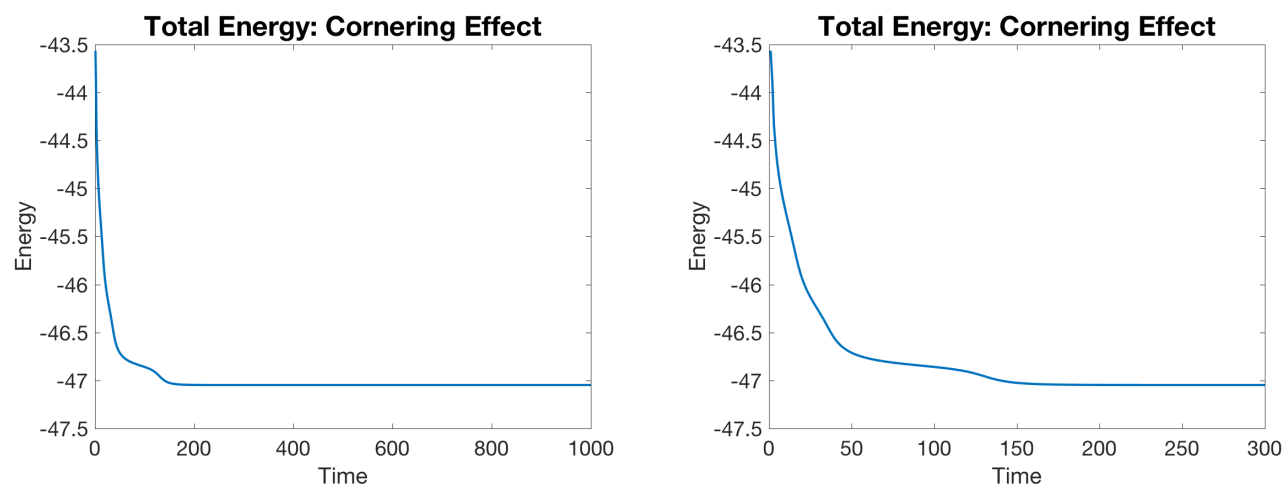

Figure 4: Total energy as a function of time for a droplet undergoing the cornering effect (Section 7.2).

\subsection{Two liquid crystal droplets colliding}

The third numerical experiment demonstrates two liquid crystal droplets colliding. The initial conditions are as follows:

$$
\begin{aligned}
& s_{h}^{0}=s^{*}, \\
& \mathbf{n}_{h}^{0}= \begin{cases}\frac{(x, y)-(0.3,0.5)}{|(x, y)-(0.3,0.5)|}, & x \leq 0.5, \\
\frac{-((x, y)-(0.7,0.5))}{|(x, y)-(0.7,0.5)|}, & x>0.5,\end{cases} \\
& \phi_{h}^{0}= \begin{cases}I_{h}\left\{-\tanh \left(\frac{(x-0.3)^{2} / 0.02+(y-0.5)^{2} / 0.02-1}{2 \varepsilon}\right)\right\}, & x \leq 0.5, \\
I_{h}\left\{-\tanh \left(\frac{(x-0.7)^{2} / 0.02+(y-0.5)^{2} / 0.02-1}{2 \varepsilon}\right)\right\}, & x>0.5 .\end{cases}
\end{aligned}
$$

The following Dirichlet boundary conditions on $\partial \Omega$ are imposed for $s$ and $\mathbf{n}$ :

$$
s=s^{*}, \quad \mathbf{n}_{h}=(1,0) \text {. }
$$

The relevant parameters are $\kappa=1, \rho=1, \omega_{\text {erk }}=1, \omega_{\text {dw }}=100, \omega_{\text {chdw }}=1, \omega_{\text {chp }}=1+\omega_{\mathrm{a}, \mathbf{n}}+$ $\omega_{\mathrm{a}, \mathrm{s}}=21, \omega_{\mathrm{a}, \mathrm{s}}=10, \omega_{\mathrm{a}, \mathbf{n}}=10$. The space step size is taken to be $h=1 / 64$ and the time step size is taken to be $\tau=0.002$ with a final stopping time of $T=2.0$. The interfacial width parameter is taken to be $\varepsilon=3 h$. Fig. 5 shows the evolution of the droplet over time. The top two rows display the evolution of the scalar degree of orientation parameter $s$. The bottom two rows show the evolution of the phase field parameter $\phi$ and the director field $\mathbf{n}$. Due to the boundary conditions for $\mathbf{n}$, the defects inside the droplets are driven to annihilate, which is what forces the droplets to merge. At equilibrium, no defects remain, because the boundary conditions for $\mathbf{n}$ are of degree zero, and the droplet takes on a lens shape.

Fig. 6 displays the energy decreasing property of the scheme for this experiment.

Droplet merging depends on the choice of weighting parameters, boundary conditions, and whether defects are present. In this example, the "sign" of the point defects in 

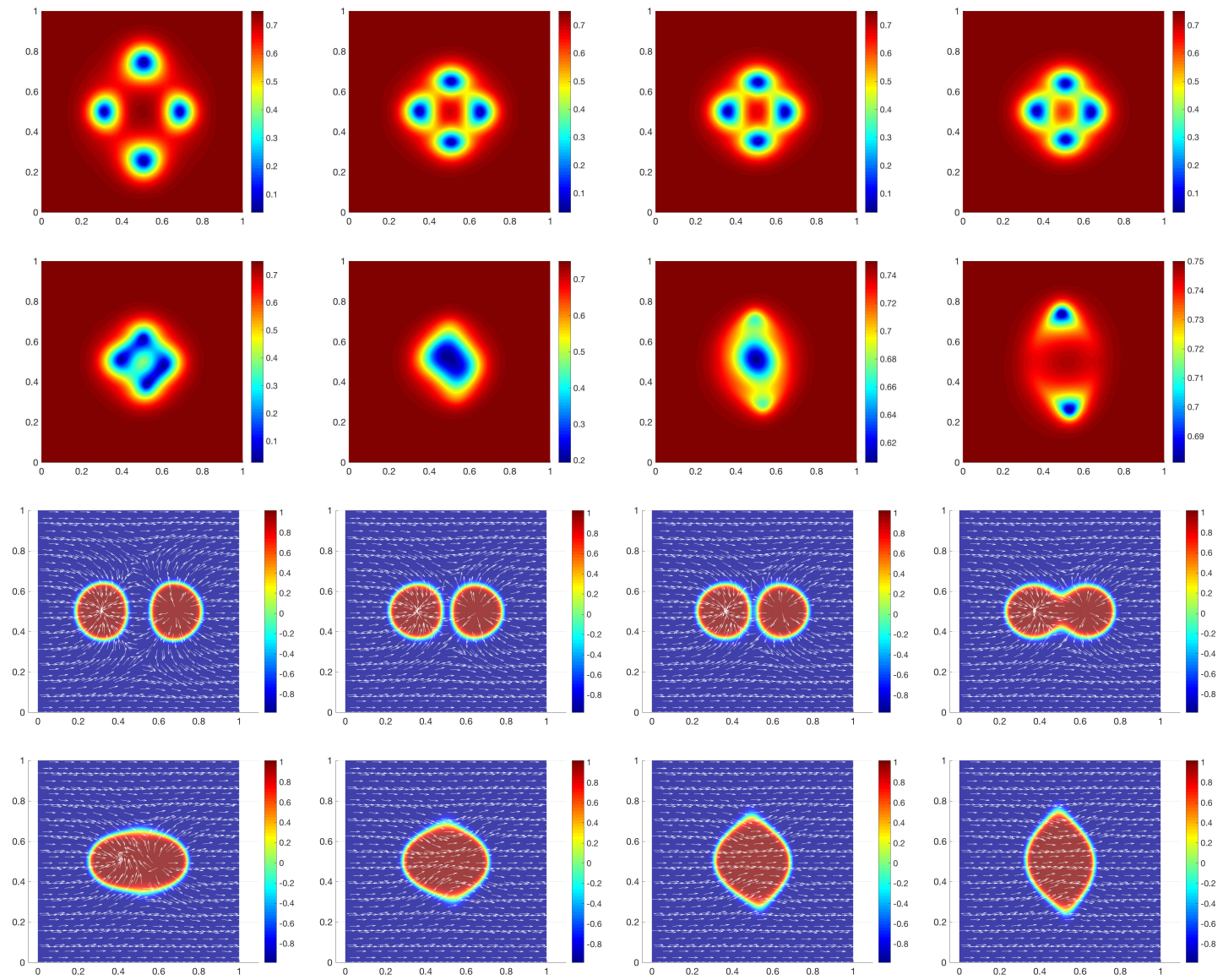

Figure 5: Droplet cornering, $\Omega=[0,1] \times[0,1], h=1 / 64, \tau=0.002$ (Section 7.3). The times displayed are $t=0.2, t=0.4, t=0.42, t=0.44$ (top from left to right) and $t=0.48, t=0.52, t=0.56, t=0.6$ (bottom from left to right).
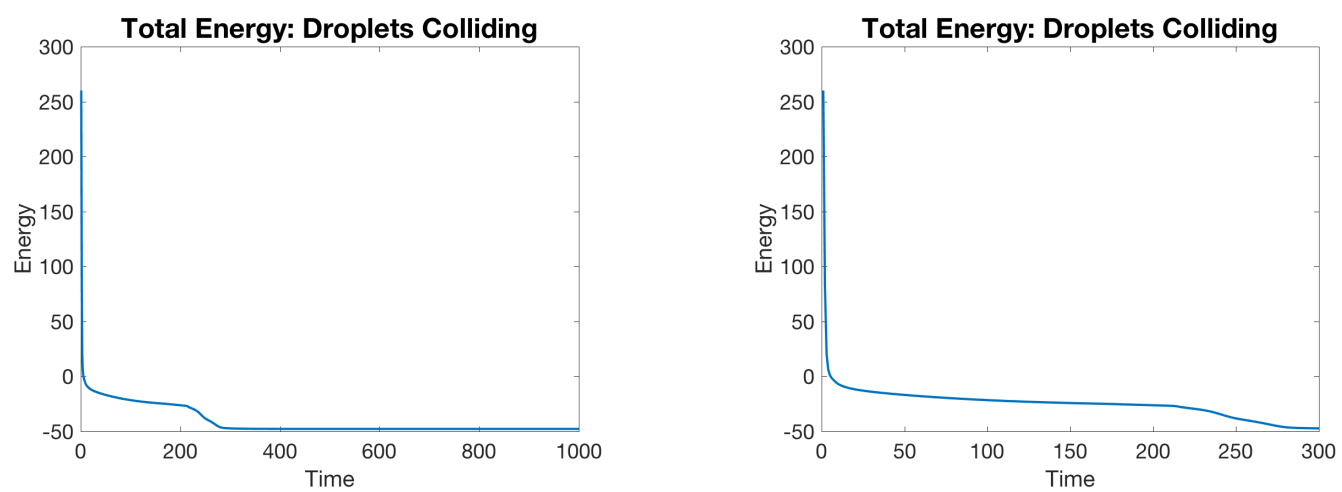

Figure 6: Total energy as a function of time for two droplets colliding (Section 7.3). 
each droplet are opposite (i.e. one defect points inside, the other points outside). If both initial point defects point outward (or inward), then this would induce a region of defect between the two droplets, i.e. where $s \approx 0$. This would prevent the droplets from merging, because the "diffuse" weak anchoring energy penalizes $s$ away from zero (recall $E_{\mathrm{a}, s}$ in (3.10)). Of course, this is related to the director field approach we use.

\subsection{A liquid crystal droplet splitting}

The fourth numerical experiment demonstrates a liquid crystal droplet splitting into two droplets. The initial conditions are as follows:

$$
\begin{aligned}
& s_{h}^{0}=s^{*}, \\
& \mathbf{n}_{h}^{0}= \begin{cases}\frac{(x, y)-(0.35,0.5)}{|(x, y)-(0.35,0.5)|}, & x \leq 0.5, \\
\frac{-((x, y)-(0.65,0.5))}{|(x, y)-(0.65,0.5)|}, & x>0.5,\end{cases} \\
& \phi_{h}^{0}=I_{h}\left\{-\tanh \left(\frac{(x-0.5)^{2} / 0.03+(y-0.5)^{2} / 0.03-1}{2 \varepsilon}\right)\right\} .
\end{aligned}
$$

The following Dirichlet boundary conditions on $\partial \Omega$ are imposed for $s$ and $\mathbf{n}$ :

$$
s=s^{*}, \quad \mathbf{n}_{h}= \begin{cases}\frac{(x, y)-(0.3,0.5)}{|(x, y)-(0.3,0.5)|}, & x \leq 0.5, \\ \frac{-((x, y)-(0.7,0.5))}{|(x, y)-(0.7,0.5)|}, & x>0.5 .\end{cases}
$$

The relevant parameters are $\kappa=1, \rho=1, \omega_{\text {erk }}=1, \omega_{\text {dw }}=100, \omega_{\text {chdw }}=1, \omega_{\text {chp }}=1+\frac{1}{4}\left(\omega_{\mathrm{a}, \mathbf{n}}+\right.$ $\left.\omega_{\mathrm{a}, \mathrm{s}}\right)=11, \omega_{\mathrm{a}, \mathrm{s}}=20, \omega_{\mathrm{a}, \mathbf{n}}=20$. The space step size is taken to be $h=1 / 64$ and the time step size is taken to be $\tau=0.002$ with a final stopping time of $T=2.0$. The interfacial width parameter is taken to be $\varepsilon=3 h$. Fig. 7 shows the evolution of the droplet over time. The top two rows display the evolution of the scalar degree of orientation parameter $s$. The bottom two rows show the evolution of the phase field parameter $\phi$ and the director field $\mathbf{n}$. The boundary conditions for $\mathbf{n}$ induce two defects in the domain with no annihilation, and the liquid crystal elastic energy acts to push the defects further apart.

Fig. 8 displays the energy decreasing property of the scheme for this experiment.

As in Section 7.3, droplet splitting depends on various factors. For instance, the weighting on the Cahn-Hilliard gradient energy term $\omega_{\mathrm{chp}}$ is lower than in the previous experiments, which effectively lowers the surface tension on the droplet. If, for example, $\omega_{\text {chp }}=1+\left(\omega_{\mathrm{a}, \mathbf{n}}+\omega_{\mathrm{a}, \mathrm{s}}\right)=21$ as before, then the droplet would hold together, and the defects would escape outside the two droplets. Since surface tension is relatively weak in this example, the droplet splits to accommodate the separation of the defects. 

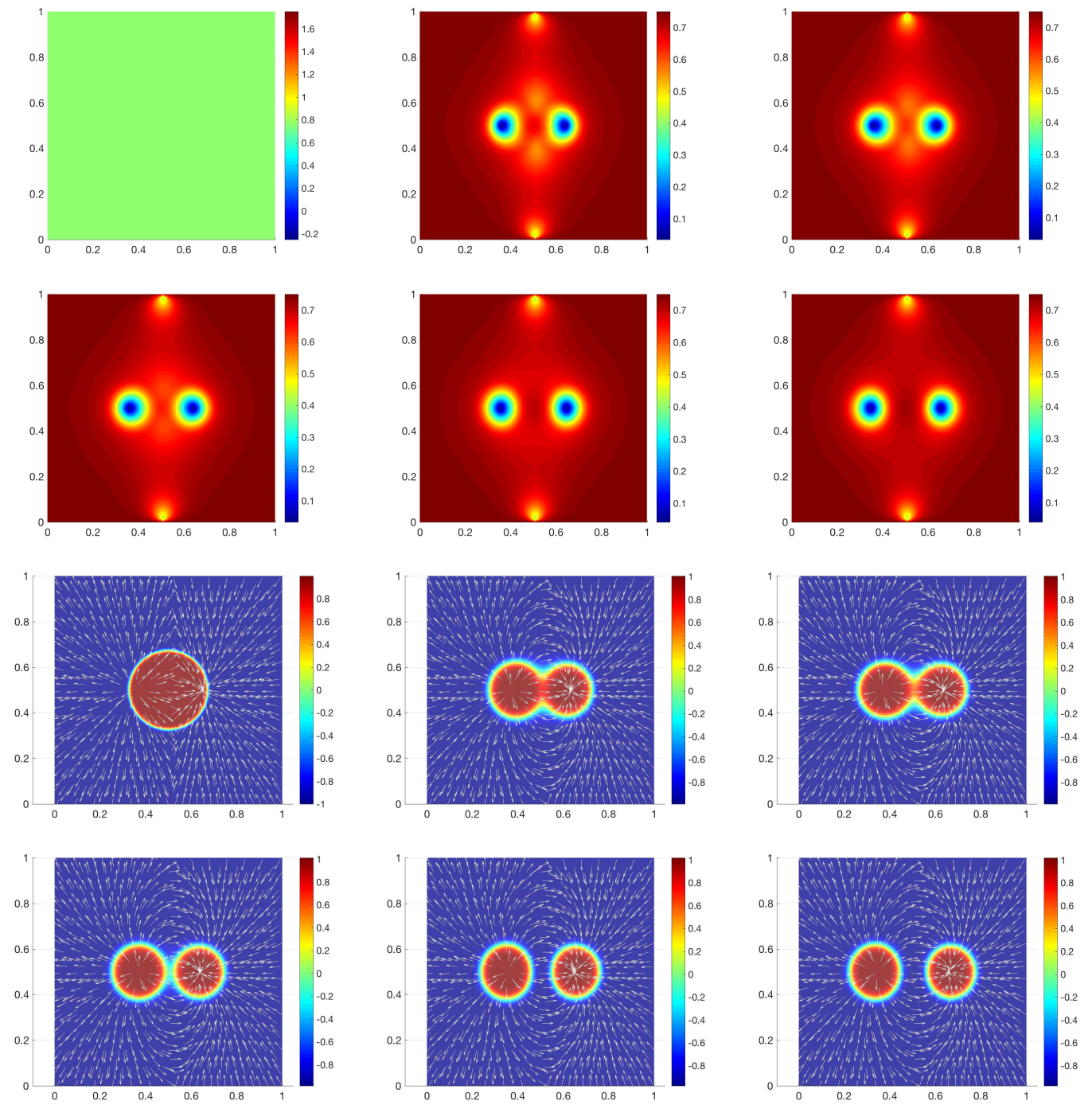

Figure 7: Droplet cornering, $\Omega=[0,1] \times[0,1], h=1 / 64, \tau=0.002$ (Section 7.4). The times displayed are $t=0, t=0.04, t=0.08$ (top from left to right) and $t=0.12, t=0.16, t=0.2$ (bottom from left to right).

\section{Conclusion}

We introduced a phase field model and finite element scheme for nematic liquid crystal droplets in a pure liquid crystal substance. We presented a finite element method and gradient flow scheme, and used it to explore gradient flow dynamics for finding energy minimizers. We were able to show that the gradient flow method has a monotone energy decreasing property. We also demonstrated that the discrete energy of the numerical scheme converges, in the sense of $\Gamma$-convergence, to the continuous free energy 

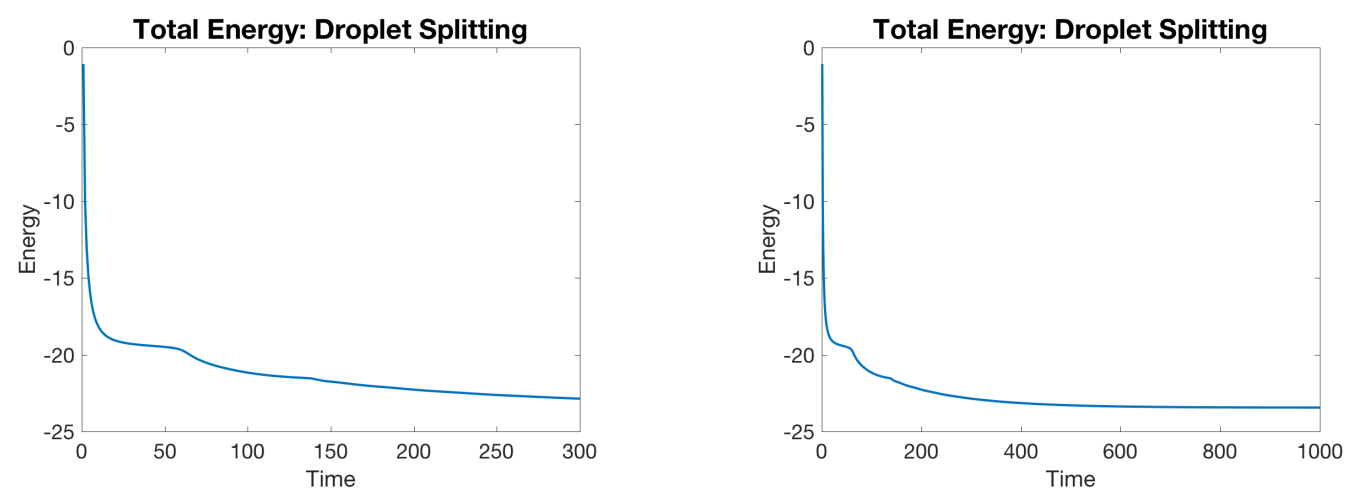

Figure 8: Total energy as a function of time for a droplet splitting (Section 7.4).

of the model. Finally, we presented numerical experiments demonstrating four different aspects of liquid crystal droplets: movement/positioning, cornering, coalescence, and splitting.

Some extensions of this work are: include more general liquid crystal elastic energies such as the consideration of an elastic constant $\kappa$ or a double well potential for the orientation parameter $f(s)$ which depends on the phase field parameter $\phi$ in order to model a liquid crystal droplet immersed in an isotropic liquid. In particular, one could consider a high contrast ratio for the constants between the two phases, so that the non-liquid crystalline material is nearly isotropic.

Other physical effects, such as electro-statics, and coupling to fluid dynamics (e.g. Stokes flow) are also of interest. Moreover, development of a multi-grid solver for the Cahn-Hilliard equation [14] would enable computations in three dimensions; indeed, this would allow for investigating the connection between defect structures and droplet shapes. Furthermore, our method could be used to model optimal shapes of liquid crystal droplets, e.g. tactoids [23], nematic droplets on fibers [6], and nematic shells [44]. Other applications could be in optimal control of droplets and self-assembly of arrays of droplets.

\section{Acknowledgments}

S. W. Walker acknowledges financial support by the NSF via DMS-1418994 and DMS1555222 (CAREER).

\section{Appendix}

In this appendix, we provide support for the formation of Eq. (2.11). We note that the details shown here are a republication of those developed in [41]. We model boundary 
conditions on the colloid's surface by imposing a diffuse weak anchoring. We note the standard form of the weak anchoring energy in the Q-tensor model [37, eqn. (66)] is

$$
J(\mathbf{Q})=\frac{K_{a}}{2} \int_{\partial \Omega_{c}}\left|\mathbf{Q}-\mathbf{Q}_{\mathbf{0}}\right|^{2},
$$

where $\mathbf{Q}_{0}$ is the desired value of $\mathbf{Q}$ on the boundary $\partial \Omega_{c}$ and $K_{a}$ is a weighting parameter. We focus on imposing homeotropic anchoring, i.e. we take $\mathbf{Q}_{0}$ to have the form of a uniaxial nematic:

$$
\mathbf{Q}_{0}=s^{*}\left(\boldsymbol{v} \otimes \boldsymbol{v}-\frac{1}{3} \mathbf{I}\right),
$$

where $v$ is the normal of $\partial \Omega_{c}$ and $s^{*}$ is the global minimum of the double well potential $f(s)$. Using the expression $\mathbf{Q}=s\left(\mathbf{n} \otimes \mathbf{n}-\frac{1}{3} \mathbf{I}\right)$ for uniaxial nematics, along with the facts that $\mathbf{Q}, \mathbf{Q}_{\mathbf{0}}$ are symmetric, $\left|\mathbf{Q}-\mathbf{Q}_{\mathbf{0}}\right|^{2}=\operatorname{tr}\left[\left(\mathbf{Q}-\mathbf{Q}_{\mathbf{0}}\right)^{2}\right]$, and $|\mathbf{n}|=|\boldsymbol{v}|=1$, a straightforward calculation gives

$$
\left|\mathbf{Q}-\mathbf{Q}_{\mathbf{0}}\right|^{2}=2 s s^{*}\left[|\mathbf{n}|^{2}|\boldsymbol{v}|^{2}-(\mathbf{n} \cdot \boldsymbol{v})^{2}\right]+\frac{2}{3}\left(s-s^{*}\right)^{2}|\boldsymbol{v}|^{2} .
$$

We use (A.3) for our "diffuse" weak anchoring in the context of the Ericksen model combined with the Cahn-Hilliard equation. In fact, noting that $v=\frac{\nabla \phi}{|\nabla \phi|}$, simplifying $s s^{*}$ with $s^{2}$, and normalizing the constants we obtain the continuous diffuse weak anchoring energy (2.11):

$$
\begin{aligned}
& E_{\mathrm{a}, \mathbf{n}}(s, \mathbf{n}, \phi)=\frac{\varepsilon}{2} \int_{\Omega} s^{2}\left[|\mathbf{n}|^{2}|\nabla \phi|^{2}-(\mathbf{n} \cdot \nabla \phi)^{2}\right] d \mathbf{x}, \\
& E_{\mathrm{a}, s}(s, \phi)=\frac{\varepsilon}{2} \int_{\Omega}|\nabla \phi|^{2}\left(s(\mathbf{x})-s^{*}\right)^{2} d \mathbf{x} .
\end{aligned}
$$

\section{References}

[1] P. J. Ackerman, J. van de Lagemaat, and I. I. Smalyukh. Self-assembly and electrostriction of arrays and chains of hopfion particles in chiral liquid crystals. Nat. Commun., 6, Jan 2015.

[2] F. Alouges. A new algorithm for computing liquid crystal stable configurations: The harmonic mapping case. SIAM J. Numer. Anal., 34(5):1708-1726, 1997.

[3] L. Ambrosio. Existence of minimal energy configurations of nematic liquid crystals with variable degree of orientation. Manuscripta Math., 68(1):215-228, 1990.

[4] T. Araki and H. Tanaka. Colloidal aggregation in a nematic liquid crystal: Topological arrest of particles by a single-stroke disclination line. Phys. Rev. Lett., 97:127801, Sep 2006.

[5] J. W. Barrett, X. Feng, and A. Prohl. Convergence of a fully discrete finite element method for a degenerate parabolic system modelling nematic liquid crystals with variable degree of orientation. ESAIM-Math. Model Num., 40:175-199, 12006.

[6] V. M. O. Batista, N. M. Silvestre, and M. M. Telo da Gama. Nematic droplets on fibers. Phys. Rev. E., 92:062507, Dec 2015. 
[7] F. Bethuel, H. Brezis, and F. Hélein. Ginzburg-Landau Vortices, Progress in Nonlinear Differential Equations and their Applications, volume 13. Birkhäuser Boston Inc., Boston, MA, 1994.

[8] H. K. Bisoyi and S. Kumar. Liquid-crystal nanoscience: an emerging avenue of soft selfassembly. Chem. Soc. Rev., 40:306-319, 2011.

[9] C. Blanc. Colloidal crystal ordering in a liquid crystal. Science, 352(6281):40-41, 2016.

[10] L.M. Blinov. Electro-optical and magneto-optical properties of liquid crystals. Wiley, 1983.

[11] A. Braides. Gamma-Convergence for Beginners, Oxford Lecture Series in Mathematics and Its Applications, volume 22. Oxford Scholarship, 2002.

[12] A. Braides. Local minimization, variational evolution and $\Gamma$-convergence. Lecture Notes in Mathematics, volume 2094. Springer, 2014.

[13] J. H. Brandts, S. Korotov, and M. Křížek. The discrete maximum principle for linear simplicial finite element approximations of a reaction-diffusion problem. Linear Algebra Appl., Special Issue in honor of Richard S. Varga, 429(10):2344 - 2357, 2008.

[14] S. C. Brenner, A. E. Diegel, and L-Y Sung. A robust solver for a mixed finite element method for the Cahn-Hilliard equation. arXiv preprint arXiv:1709.04003, 2017.

[15] H. Brezis, J-M Coron, and E. H. Lieb. Harmonic maps with defects. Commun. Math. Phys., 107(4):649-705, 1986.

[16] J. W. Cahn. On spinodal decomposition. Acta Metall, 9:795, 1961.

[17] J. W. Cahn and J. E. Hilliard. Free energy of a nonuniform system. I. interfacial free energy. J. Chem. Phys, 28:258, 1958.

[18] M. Calderer, D. Golovaty, F. Lin, and C. Liu. Time evolution of nematic liquid crystals with variable degree of orientation. SIAM J. Math. Anal., 33(5):1033-1047, 2002.

[19] P. G. Ciarlet and P. A. Raviart. Maximum principle and uniform convergence for the finite element method. Comput. Method Appl. M., 2(1):17 - 31, 1973.

[20] R. Cohen, S-Y Lin, and M Luskin. Relaxation and gradient methods for molecular orientation in liquid crystals. Comput. Phys. Commun., 53(1-3):455 - 465, 1989.

[21] G. Dal Maso. An introduction to $\Gamma$-convergence. Progress in Nonlinear Differential Equations and their Applications, 8. Birkhäuser Boston, Inc., Boston, MA, 1993.

[22] P. G. de Gennes and J. Prost. The Physics of Liquid Crystals, International Series of Monographs on Physics, volume 83. Oxford Science Publication, Oxford, UK, 2nd edition, 1995.

[23] A. DeBenedictis and T. J. Atherton. Shape minimisation problems in liquid crystals. Liq. Cryst., 43(13-15):2352-2362, 2016.

[24] A. E. Diegel, X. Feng, and S. M. Wise. Analysis of a mixed finite element method for a Cahn-Hilliard-Darcy-Stokes system. SIAM J. Numer. Anal., 53(1):127-152, 2015.

[25] J. L. Ericksen. Liquid crystals with variable degree of orientation. Arch. Rational Mech. An., 113(2):97-120, 1991.

[26] L. C. Evans. Partial Differential Equations. American Mathematical Society, Providence, Rhode Island, 1998.

[27] C. Gugenberger, R. Spatschek, and K. Kassner. Comparison of phase-field models for surface diffusion. Phys. Rev. E., 78:016703, Jul 2008.

[28] F. M. Guillén González and J. V. Gutiérrez-Santacreu. A linear mixed finite element scheme for a nematic Ericksen- Leslie liquid crystal model. ESAIM-Math. Model. Num., 47:1433-1464, 92013.

[29] M. Humar and I. Muševič. 3d microlasers from self-assembled cholesteric liquid-crystal microdroplets. Opt. Express, 18(26):26995-27003, Dec 2010.

[30] S. Korotov, M. Kř́̌žek, and P. Neittaanmäkia. Weakened acute type condition for tetrahedral 
triangulations and the discrete maximum principle. Math. Comput., 70(233):107-119, January 2001.

[31] J. P. F. Lagerwall and G. Scalia. A new era for liquid crystal research: Applications of liquid crystals in soft matter nano-, bio- and microtechnology. Curr. Appl. Phys., 12(6):1387 - 1412, 2012.

[32] F-H Lin. Nonlinear theory of defects in nematic liquid crystals; phase transition and flow phenomena. Commun. Pur. Appl. Math., 42(6):789-814, 1989.

[33] F-H Lin. On nematic liquid crystals with variable degree of orientation. Commun. Pur. Appl. Math., 44(4):453-468, 1991.

[34] S-Y Lin and M. Luskin. Relaxation methods for liquid crystal problems. SIAM J. Numer. Anal., 26(6):1310-1324, 1989.

[35] C. Liu and N. Walkington. Approximation of liquid crystal flows. SIAM J. Numer. Anal., 37(3):725-741, 2000.

[36] J. A. Moreno-Razo, E. J. Sambriski, N. L. Abbott, J. P. Hernández-Ortiz, and J. J. de Pablo. Liquid-crystal-mediated self-assembly at nanodroplet interfaces. Nature, 485(7396):86-89, May 2012.

[37] Nigel J Mottram and Christopher JP Newton. Introduction to q-tensor theory. arXiv preprint arXiv:1409.3542, 2014.

[38] I. Muševič and S. Žumer. Liquid crystals: Maximizing memory. Nat. Mater., 10(4):266-268, April 2011.

[39] R. H. Nochetto, S. W. Walker, and W. Zhang. Numerics for liquid crystals with variable degree of orientation. In Symposium NN - Mathematical and Computational Aspects of Materials Science, volume 1753 of MRS Proceedings, 2015.

[40] R. H. Nochetto, S. W. Walker, and W. Zhang. A finite element method for nematic liquid crystals with variable degree of orientation. SIAM J. Numer. Anal., 55(3):1357-1386, 2017.

[41] R. H. Nochetto, S. W. Walker, and W. Zhang. The Ericksen model of liquid crystals with colloidal and electric effects. J. Comput. Phys., 352:568-601, 2018.

[42] M. Rahimi, T. F. Roberts, J. C. Armas-Prez, X. Wang, E. Bukusoglu, N. L. Abbott, and J. J. de Pablo. Nanoparticle self-assembly at the interface of liquid crystal droplets. P. Natl. Acad. Sci. USA, 112(17):5297-5302, 2015.

[43] R. Schoen and K. Uhlenbeck. A regularity theory for harmonic maps. J. Differ. Geom., 17(2):307-335, 1982.

[44] F. Serra. Curvature and defects in nematic liquid crystals. Liq. Cryst., 43(13-15):1920-1936, 2016.

[45] A. A. Shah, H. Kang, K. L. Kohlstedt, K. H. Ahn, S. C. Glotzer, C. W. Monroe, and M. J. Solomon. Self-assembly: Liquid crystal order in colloidal suspensions of spheroidal particles by direct current electric field assembly (small 10/2012). Small, 8(10):1457-1457, 2012.

[46] J. Shen and X. Yang. Numerical approximations of Allen-Cahn and Cahn-Hilliard equations. Discrete Contin. Dyn. Syst., 28(4):1669 - 1691, 2010.

[47] J. Shen and X. Yang. A phase-field model and its numerical approximation for two-phase incompressible flows with different densities and viscosities. SIAM J. Sci. Comput., 32(3):1159$1179,2010$.

[48] G. Strang and G. Fix. An Analysis of the Finite Element Method. Wellesley-Cambridge, 2nd edition, May 2008.

[49] J. Sun, H. Wang, L. Wang, H. Cao, H. Xie, X. Luo, J. Xiao, H. Ding, Z. Yang, and H. Yang. Preparation and thermo-optical characteristics of a smart polymer-stabilized liquid crystal thin film based on smectic Achiral nematic phase transition. Smar. Mat. and St., 
23(12):125038, 2014.

[50] J. E. Taylor and J. W. Cahn. Diffuse interfaces with sharp corners and facets: phase field models with strongly anisotropic surfaces. Physica D., 112(3-4):381-411, 1998.

[51] S. Torabi, J. Lowengrub, A. Voigt, and S. Wise. A new phase-field model for strongly anisotropic systems. P. Roy Soc. of Lond. A Mat., 2009.

[52] E. G. Virga. Variational Theories for Liquid Crystals, volume 8. Chapman and Hall, London, 1st edition, 1994.

[53] S. W. Walker. Felicity wiki documentation, 2017.

[54] N. J. Walkington. Numerical approximation of nematic liquid crystal flows governed by the Ericksen-Leslie equations. ESAIM-Math. Model Num., 45:523-540, 52011.

[55] M. Wang, L. He, S. Zorba, and Y. Yin. Magnetically actuated liquid crystals. Nano Lett., 14(7):3966-3971, 2014. PMID: 24914876.

[56] S. M. Wise, C. Wang, and J. S. Lowengrub. An energy-stable and convergent finite-difference scheme for the phase field crystal equation. SIAM J. Numer. Anal., 47(3):2269-2288, June 2009.

[57] X. Yang, J. Zhao, and Q. Wang. Numerical approximations for the molecular beam epitaxial growth model based on the invariant energy quadratization method. J. Comput. Phys., 333:104-127, 2017.

[58] J. Zhao, H. Li, Q. Wang, and X. Yang. Decoupled energy stable schemes for a phase field model of three-phase incompressible viscous fluid flow. J. Sci. Comput., 70(3):1367-1389, 2017.

[59] J. Zhao and Q. Wang. Semi-discrete energy-stable schemes for a tensor-based hydrodynamic model of nematic liquid crystal flows. J. Sci. Comput., 68(3):1241-1266, 2016.

[60] J. Zhao, Q. Wang, and X. Yang. Numerical approximations to a new phase field model for two phase flows of complex fluids. Comput. Method Appl. M., 310:77-97, 2016.

[61] J. Zhao, X. Yang, Y. Gong, and Q. Wang. A novel linear second order unconditionally energy stable scheme for a hydrodynamic-tensor model of liquid crystals. Comput. Method Appl. M., 318:803-825, 2017.

[62] J. Zhao, X. Yang, J. Shen, and Q. Wang. A decoupled energy stable scheme for a hydrodynamic phase-field model of mixtures of nematic liquid crystals and viscous fluids. J. Comput. Phys., 305:539-556, 2016. 